Bull. Chem. Soc. Ethiop. 2021, 35(1), 61-76.

(C) 2021 Chemical Society of Ethiopia and The Authors

ISSN 1011-3924

DOI: https://dx.doi.org/10.4314/bcse.v35i1.5

Printed in Ethiopia

Online ISSN 1726-801X

\title{
SYNTHESIS, SPECTRAL AND SOL-GEL BEHAVIOR OF MIXED LIGAND COMPLEXES OF TITANIUM(IV) WITH OXYGEN, NITROGEN AND SULFUR DONOR LIGANDS
}

\author{
Abhishek Srivastava $^{1}$, Neetu Srivastava ${ }^{2}$ and Umesh Nath Tripathi ${ }^{2}$ \\ ${ }^{1}$ Department of Chemistry, GLA University, Mathura, U.P., India \\ ${ }^{2}$ Department of Chemistry, D.D.U. Gorakhpur University, Gorakhpur, 273001, U.P., India
}

(Received November 28, 2019; Revised January 21, 2021; Accepted January 25, 2021)

\begin{abstract}
A new route to synthesize nano-sized Ti(IV) mixed ligand complexes have been investigated by the reaction of titanium(IV) chloride with ammonium salts of dithiophosphate and 3(2'-hydroxyphenyl)-5-(4substituted phenyl) pyrazolines. The resultant complex is then treated with $\mathrm{H}_{2} \mathrm{~S}$ gas to get sulfur bridged dimer of $\mathrm{Ti}(\mathrm{IV})$ complex, a precursor of $\mathrm{TiS}_{2}$. The morphology of the complexes was studied by employing XRD which shows that all the complexes are amorphous solid. Molecular weight measurements, elemental analysis in conjugation with spectroscopic (IR, ${ }^{1} \mathrm{H}$ NMR, ${ }^{13} \mathrm{C}$ NMR and ${ }^{31} \mathrm{P} \mathrm{NMR}$ ) studies revealed the dimeric nature of the complexes in which pyrazoline and dithiophosphate are bidentate. Scanning electron microscopic image and XRD indicate that the particles are in the nano range $(50 \mathrm{~nm})$. Putting all the facts together, coordination number six is proposed for titanium with octahedral geometry.
\end{abstract}

KEY WORDS: Titanium(IV), Dithiophosphate, Pyrazoline, Nano-sized, Sol-gel, Mixed ligand complexes

\section{INTRODUCTION}

Titanium proves to be an excellent corrosion-resistance material in many environments as it forms a protective oxide layer on its surface $[1,2]$. The high tensile strength, light weight and excellent corrosion resistant make the titanium a useful alloying agent for many parts of highspeed aircraft, motorbikes, ships and missiles [3-5]. Titanium being a biocompatible material found application in prosthetic devices $[6,7]$.

The Ti(IV) complexes with nitrogen, oxygen and sulfur donor ligands have received considerable attention due to their widespread utilization as an active precursor for making $\mathrm{TiO}_{2}$ and $\mathrm{TiS}_{2}[8]$. Owing to the hard acid character of titanium, the synthesis of its simple thiolates was not possible. Attempts have been made to reduce the acidic strength of titanium metal centre by attaching electron-rich ligands such as dialkyl nitrogen and cyclopentadienyl which then forms a stable complex with soft bases [9]. The highly sensitive nature of titanium complexes towards hydrolysis reduces its activity towards different applications [10]. Available reports showed that the addition of bulky electron-rich ligands to Ti metal centre increases the resistance of metal complexes towards hydrolysis [11,12].

The excellent biological activity of sulfur containing transition metal complexes makes them interesting [13]. Several reports are available on alkylene and O,O'-dialkyl dithiophosphate derivatives of $\mathrm{Ag}(\mathrm{I}), \mathrm{Zr}(\mathrm{IV}), \mathrm{Fe}(\mathrm{II})$ and $\mathrm{Cu}(\mathrm{II})$ [14, 15]. Carmalt et al. [9] reported titanium pyridine and pyridine thiolates as a precursor for the production of titanium disulfide. Ti(IV) has been extensively used for the polymerization of ethylene and propylene [16, 17]. Salen-Ti(IV) complex has been effectively employed in the controlled polymerization of D,L-lactic acid [18]. Park et al. [19] designed and synthesized a new class of green colored titanium complexes with a dithiolate ligand for LCD and TFT panels. The first non-platinum anticancer drug exhibiting excellent efficacy was titanium based titanocene dichloride and budotitane [20]. Later on,

*Corresponding author. E-mail: aabhichem@gla.ac.in

This work is licensed under the Creative Commons Attribution 4.0 International License 
numbers of complexes of titanium have been reported by several researchers which can act as a potent cytotoxic agent to kill cancer cells through apoptosis [21-24].

Several reports are available on titanium complexes of thiolates, dithiolates and dithiocarbamates [25-29]. The limited study on mixed ligand complexes of Ti(IV) in general and with pyrzoline $[21-24,30]$ and dithiophosphate in particular [31], furthermore the wide range application of nanosized $\mathrm{TiS}_{2}$ [32-35] drawn our attention to develop new series of Ti(IV) complexes with dithiophosphate and pyrazoline ligands applying sol-gel method. The resulting complex may act as a precursor for nano $\mathrm{TiS}_{2}$.

\section{Materials and methods}

\section{EXPERIMENTAL}

Absolutely dry conditions were maintained throughout the reaction process as titanium tetrachloride reacts violently with water to produce toxic and highly corrosive $\mathrm{HCl}$ gas. Standard procedures were used to distill, purify and dry the solvents [36]. Phosphorus pentasulfide (s.d. fine chemicals, Mumbai), titanium tetrachloride (E. Merck), O-hydroxy acetophenone (CDH), benzaldehydes (E. Merk), sodium hydroxide (Glaxo), hydrochloric acid (Ranbaxy), acetic acid $(\mathrm{CDH})$, and hydrazine hydrate (Ranbaxy) were used as received without any further purification. Pyrazolines and ammonium salt of dialkyl/alkylene dithiophosphates were prepared by the literature method [37, 38]. Titanium was estimated gravimetrically by cupferron's method while chlorine by Volhard's method. Perkin Elmer (2400 Series II) CHNS/O analyzer was used for the elemental analysis (C, H, N and S). IR Spectra were recorded in the range of $4000-200 \mathrm{~cm}^{-1}$ on the Varian 3100 FT-IR spectrophotometer. Proton decoupled NMR spectra $\left({ }^{13} \mathrm{C},{ }^{1} \mathrm{H}\right.$ NMR) were recorded (room temperature) on JOEL AL 300 FT NMR spectrophotometer at an operating frequency of $300.40 \mathrm{MHz}$. Bruker Nonious Kappa CCD diffractometer was used for X-ray diffraction studies. The FAB mass spectra were recorded on JOEL SX102 mass spectrometer using Argon or Xenon $(6 \mathrm{kV}, 10 \mathrm{~mA})$ as the FAB gas.

\section{Synthesis of substituted dithiophosphate ligands}

O,O'-Dialkyl and alkylene dithiophosphoric acids were synthesized by reacting phosphorus pentasulfide with the corresponding phenols or alcohols (1:4 molar ratio) and with glycols $(1: 2$ molar ratio) as shown in the following chemical reactions (Eq. 1-2).

$$
\mathrm{P}_{2} \mathrm{~S}_{5}+4 \mathrm{ROH} \frac{\text { Dry Benzene, } 50^{\circ} \mathrm{C}}{8-10 \text { hour Stirring }} \quad 2\left[(\mathrm{OR})_{2} \mathrm{P}(\mathrm{S}) \mathrm{S}\right] \mathrm{H}+\mathrm{H}_{2} \mathrm{~S} \uparrow
$$

where $\mathrm{R}=-\mathrm{C}_{6} \mathrm{H}_{5}$ or $-\mathrm{CH}_{2} \mathrm{CH}_{2} \mathrm{CH}_{3}$

$$
\mathrm{P}_{2} \mathrm{~S}_{5}+2 \mathrm{G}(\mathrm{OH})_{2} \quad \frac{\text { Dry Benzene, } 50^{\circ} \mathrm{C}}{8-10 \text { hour Stirring }}-2\left[\mathrm{O}_{2} \mathrm{GP}(\mathrm{S}) \mathrm{S}\right] \mathrm{H}+\mathrm{H}_{2} \mathrm{~S} \uparrow
$$

where $\mathrm{G}=-\mathrm{C}\left(\mathrm{CH}_{3}\right)_{2} \mathrm{C}\left(\mathrm{CH}_{3}\right)_{2},-\mathrm{CH}_{2} \mathrm{C}\left(\mathrm{C}_{2} \mathrm{H}_{5}\right)_{2} \mathrm{CH}_{2}-,-\mathrm{CH}_{2} \mathrm{CH}_{2} \mathrm{CH}\left(\mathrm{CH}_{3}\right),-\mathrm{C}\left(\mathrm{CH}_{3}\right)_{2} \mathrm{CH}_{2} \mathrm{CH}\left(\mathrm{CH}_{3}\right)-$, $-\mathrm{CH}_{2} \mathrm{C}\left(\mathrm{CH}_{3}\right)_{2} \mathrm{CH}_{2}$ - and $-\mathrm{CH}\left(\mathrm{CH}_{3}\right) \mathrm{CH}\left(\mathrm{CH}_{3}\right)-$.

The corresponding ammonium salts of the synthesized dithiophosphoric acids have been prepared by passing dry ammonia gas through their benzene solutions (Eq. 3-4). The structure of ammonium salt of substituted dithiophosphate ligands are shown in Figure 1.

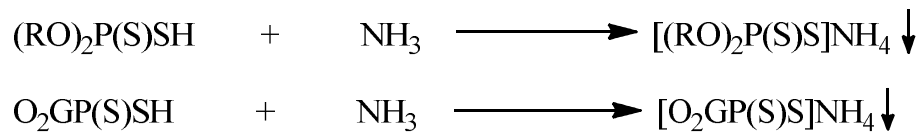

Bull. Chem. Soc. Ethiop. 2021, 35(1) 

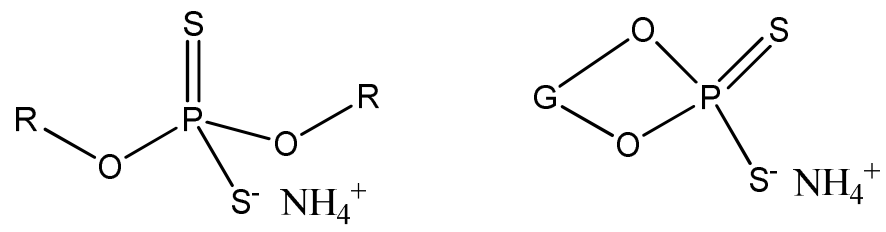

Figure 1. Structure of ammonium salt of substituted dithiophosphate ligands.

Synthesis of substituted pyrazoline ligands

Substituted pyrazoline ligands were synthesized by reported procedure [38].

(a) Synthesis of substituted 2'-hydroxychalcone. A hot solution of sodium hydroxide was added to a mixture of $o$-hydroxyacetophenone and substituted benzaldehyde in ethanol. The mixture was stirred at room temperature for 6-8 hours. The sodium salt of the chalcone was obtained as dark yellow thick mass. It was cooled in ice and neutralized with aqueous acetic acid $(50 \%)$. The yellow solid separated was filtered and washed with water before drying. Crystallization from ethanol yielded yellow needles.

(b) Synthesis of substituted pyrazoline. A mixture of substituted 2'-hydroxychalcone and hydrazine hydrate in ethanol was refluxed for 3-4 hours. It was allowed to cool at room temperature. A white crystalline solid thus obtained was separated, washed with water and dried. Recrystallization with ethanol afforded white crystals of pyrazoline. The structure of substituted pyrazoline ligand is shown in Figure 2.

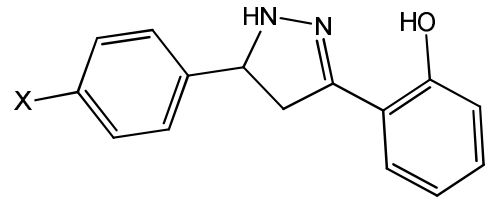

Figure 2. Structure of substituted pyrazoline.

Synthesis of $\mathrm{TiCl}_{2}\left(\mathrm{C}_{15} \mathrm{H}_{12} \mathrm{~N}_{2} \mathrm{OX}\right)(\mathrm{RO})_{2} \mathrm{PS}_{2}$

A benzene solution of pyrazoline $(1.21 \mathrm{~g}, 5.10 \mathrm{mmol})$ was added dropwise with constant stirring to the titanium tetrachloride $(0.96 \mathrm{~g}, 5.11 \mathrm{mmol})$ suspension at room temperature. To ensure the completion of reaction, the reaction mixture was stirred for 2-3 hours. To the above reaction mixture, the solution of ammonium salt of dithiophosphate in methanol was added dropwise under constant stirring for 3-4 hours. The by-product $\left(\mathrm{NH}_{4} \mathrm{Cl}\right)$ was filtered off using alkoxy funnel. A reddish-brown solid compound was obtained $(1.76 \mathrm{~g}, 88 \%)$ after removal of the volatiles from the filtrate under reduced pressure. The same procedure was adopted for the synthesis of all the compounds (2-24).

The two-step reaction scheme is proposed for the synthesis of mixed ligand titanium complexes of the general formula $\left.\mathrm{TiCl}_{2}\left(\mathrm{C}_{15} \mathrm{H}_{12} \mathrm{~N}_{2} \mathrm{OX}\right)(\mathrm{RO})_{2} \mathrm{PS}_{2}\right]$ (Eq. 5-6).

Step 1: (1:1 molar ratio)

$\mathrm{TiCl}_{4}+\mathrm{C}_{15} \mathrm{H}_{13} \mathrm{~N}_{2} \mathrm{OX} \underset{2-3 \text { hous Stirring }}{\stackrel{\text { Benzene }}{\longrightarrow}} \mathrm{TiCl}_{3}\left(\mathrm{C}_{15} \mathrm{H}_{12} \mathrm{~N}_{2} \mathrm{OX}\right)+\mathrm{HCl} \uparrow$ 
Step 2: (1:1 molar ratio)

$\begin{aligned} \mathrm{TiCl}_{3}\left(\mathrm{C}_{15} \mathrm{H}_{12} \mathrm{~N}_{2} \mathrm{OX}\right)+\left[(\mathrm{RO})_{2} \mathrm{PS}_{2}\right] \mathrm{NH}_{4} \underset{3-4 \text { hour Stirring }}{\stackrel{\text { Methanol/benzene }}{\longrightarrow}} & \mathrm{TiCl}_{2}\left(\mathrm{C}_{15} \mathrm{H}_{12} \mathrm{~N}_{2} \mathrm{OX}\right)(\mathrm{RO})_{2} \mathrm{PS}_{2} \\ & +\mathrm{NH}_{4} \mathrm{Cl} \downarrow\end{aligned}$

Synthesis of $\mathrm{Ti}_{2}\left(\mathrm{C}_{15} \mathrm{H}_{12} \mathrm{~N}_{2} \mathrm{OX}\right)_{2}\left[(\mathrm{RO})_{4} \mathrm{P}_{2} \mathrm{~S}_{6}\right]$

In the methanolic solution of $\left[\mathrm{TiCl}_{2}\left(\mathrm{C}_{15} \mathrm{H}_{12} \mathrm{~N}_{2} \mathrm{OX}\right)(\mathrm{RO})_{2} \mathrm{PS}_{2}\right]$, dry $\mathrm{H}_{2} \mathrm{~S}$ gas was passed for 1-2 hours which result in the formation of orange-colored precipitate (Eq. 7-8).

$$
\begin{aligned}
& \mathrm{TiCl}_{2}\left(\mathrm{C}_{15} \mathrm{H}_{12} \mathrm{~N}_{2} \mathrm{OX}\right)(\mathrm{RO})_{2} \mathrm{PS}_{2}+\mathrm{H}_{2} \mathrm{~S} \text { (excess) } \stackrel{\text { Methanol }}{\longrightarrow} \mathrm{Ti}_{2}\left(\mathrm{C}_{15} \mathrm{H}_{12} \mathrm{~N}_{2} \mathrm{OX}\right)_{2}\left[(\mathrm{RO})_{4} \mathrm{P}_{2} \mathrm{~S}_{6}\right] \\
& \mathrm{TiCl}_{2}\left(\mathrm{C}_{15} \mathrm{H}_{12} \mathrm{~N}_{2} \mathrm{OX}\right)\left(\mathrm{O}_{2} \mathrm{GPS}_{2}\right)+\mathrm{H}_{2} \mathrm{~S} \text { (excess) } \stackrel{\text { Methanol }}{\longrightarrow} \mathrm{Ti}_{2}\left(\mathrm{C}_{15} \mathrm{H}_{12} \mathrm{~N}_{2} \mathrm{OX}\right)_{2}\left(\mathrm{O}_{2} \mathrm{G}_{2} \mathrm{P}_{2} \mathrm{~S}_{6}\right.
\end{aligned}
$$

where $\mathrm{X}=-\mathrm{H},-\mathrm{CH}_{3},-\mathrm{OCH}_{3},-\mathrm{Cl} ; \mathrm{R}=-\mathrm{CH}_{2} \mathrm{CH}_{2} \mathrm{CH}_{3},-\mathrm{C}_{6} \mathrm{H}_{5} ; \mathrm{G}=-\mathrm{CH}_{2} \mathrm{CMe}_{2} \mathrm{CH}_{2},-\mathrm{CMe}_{2} \mathrm{CMe}_{2}-$, $\mathrm{CH}_{2} \mathrm{CH}_{2} \mathrm{CHMe}$-.

\section{RESULTS AND DISCUSSION}

All the synthesized compounds are non-hygroscopic orange-colored solid which are stable at room temperature. They are easily soluble in coordinating solvents (THF, DMSO and DMF) as well as in common organic solvents (benzene, chloroform and methanol). The proposed stoichiometries of the synthesized compounds are in good agreement with the elemental analysis $(\mathrm{H}, \mathrm{C}, \mathrm{N}, \mathrm{S}, \mathrm{Cl}$, and $\mathrm{Ti})$ data reported in Table 1.

Spectral analysis of $\mathrm{Ti}_{2}\left(\mathrm{C}_{15} \mathrm{H}_{12} \mathrm{~N}_{2} \mathrm{OX}\right)_{2}\left[(\mathrm{RO})_{4} \mathrm{P}_{2} \mathrm{~S}_{6}\right]$

\section{Infrared spectral data analysis}

The medium intensity band observed at $3346-3325 \mathrm{~cm}^{-1}$ could be assigned to vibrations corresponding to $v[\mathrm{~N}-\mathrm{H}]$ stretching [39] while the spectral bands in the region 1624-1604 $\mathrm{cm}^{-1}$ are due to the $v[\mathrm{C}=\mathrm{N}]$ stretching vibration [40]. As compared to free pyrazoline the $v[\mathrm{C}=\mathrm{N}]$ stretching in all the synthesized compounds is observed to be shifted to the lower wavenumber. This suggests that the imino nitrogen of pyrazoline is coordinated to a metal centre. The complete absence of a signal at $\sim 3080 \mathrm{~cm}^{-1}$ in synthesized metal complexes, which is due to $v(\mathrm{O}-\mathrm{H})$ stretching originally present in pyrazoline ligands suggests that the oxygen is covalently bonded to Ti metal. This is further confirmed by the appearance of the band in the region 485$460 \mathrm{~cm}^{-1}$ corresponding to $v$ [Ti-O] stretching vibration. The bands present in $824-899 \mathrm{~cm}^{-1}$ and $1078-1050 \mathrm{~cm}^{-1}$ region has been assigned respectively to $v[\mathrm{P}-\mathrm{O}-(\mathrm{C})][41,42]$ and $v[(\mathrm{P})-\mathrm{O}-\mathrm{C}]$ $[43,44]$. The new bands of medium intensity observed in the region $549-529 \mathrm{~cm}^{-1}$ may be assigned to $v[\mathrm{P}-\mathrm{S}]$ stretching modes [45].

In comparison to free ligands, the appearance of two new bands in $335-321 \mathrm{~cm}^{-1}$ and $302-$ $290 \mathrm{~cm}^{-1}$ region corresponds to $v[\mathrm{Ti}-\mathrm{S}]$ stretching vibrations. Splitting of bands into two regions indicates that two types of sulfur are present in the molecule, one is terminal sulfur and another is bridging sulfur. The appearance of bands in the region $396-380 \mathrm{~cm}^{-1}$ has been ascribed to vibrations corresponding to $v[\mathrm{Ti}-\mathrm{N}]$ stretching [46]. The IR data of synthesized complexes are compiled in Table 2. 
Table 1. Analytical data for $\mathrm{Ti}_{2}\left(\mathrm{C}_{15} \mathrm{H}_{12} \mathrm{~N}_{2} \mathrm{OH}\right)_{2}\left[\mathrm{~S}_{6} \mathrm{P}_{2}\left(\mathrm{OCH}_{2} \mathrm{CH}_{2} \mathrm{CH}_{3}\right)_{4}\right]$.

\begin{tabular}{|c|c|c|c|c|c|c|c|c|}
\hline \multirow{2}{*}{$\begin{array}{l}\text { S. } \\
\text { No. }\end{array}$} & \multirow{2}{*}{ Compound } & \multirow{2}{*}{$\begin{array}{c}\text { Mol. Wt. } \\
\text { found } \\
\text { (calculated } \\
\text { ) }\end{array}$} & & \multicolumn{5}{|c|}{$\begin{array}{l}\text { Analysis found } \\
\text { (calculated) }\end{array}$} \\
\hline & & & Metal & $\mathrm{S}$ & $\bar{C}$ & $\mathrm{~N}$ & H & $\mathrm{Cl}$ \\
\hline 1 & $\mathrm{Ti}_{2}\left(\mathrm{C}_{15} \mathrm{H}_{12} \mathrm{~N}_{2} \mathrm{OH}\right)_{2}\left[\mathrm{~S}_{6} \mathrm{P}_{2}\left(\mathrm{OCH}_{2} \mathrm{CH}_{2} \mathrm{CH}_{3}\right)_{4}\right]$ & $\begin{array}{c}1055 \\
(1059.8)\end{array}$ & $\begin{array}{c}9.09 \\
(9.04)\end{array}$ & $\begin{array}{c}18.06 \\
(18.12)\end{array}$ & $\begin{array}{c}47.49 \\
(47.56)\end{array}$ & $\begin{array}{c}5.36 \\
(5.28)\end{array}$ & $\begin{array}{c}5.02 \\
(5.09)\end{array}$ & - \\
\hline 2 & $\mathrm{Ti}_{2}\left(\mathrm{C}_{15} \mathrm{H}_{12} \mathrm{~N}_{2} \mathrm{OH}\right)_{2}\left[\mathrm{~S}_{6} \mathrm{P}_{2}\left(\mathrm{OC}_{6} \mathrm{H}_{5}\right)_{4}\right]$ & $\begin{array}{c}1195 \\
(1195.8)\end{array}$ & $\begin{array}{c}8.08 \\
(8.01)\end{array}$ & $\begin{array}{c}16.02 \\
(16.06)\end{array}$ & $\begin{array}{c}54.24 \\
(54.19)\end{array}$ & $\begin{array}{c}4.65 \\
(4.68)\end{array}$ & $\begin{array}{c}3.82 \\
(3.85)\end{array}$ & - \\
\hline 3 & 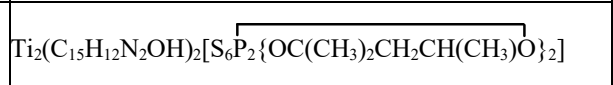 & $\begin{array}{c}1059 \\
(1055.8)\end{array}$ & $\begin{array}{c}9.03 \\
(9.07)\end{array}$ & $\begin{array}{c}18.16 \\
(18.19)\end{array}$ & $\begin{array}{c}47.69 \\
(47.74)\end{array}$ & $\begin{array}{c}5.27 \\
(5.30)\end{array}$ & $\begin{array}{c}4.72 \\
(4.74)\end{array}$ & - \\
\hline 4 & $\mathrm{Ti}_{2}\left(\mathrm{C}_{15} \mathrm{H}_{12} \mathrm{~N}_{2} \mathrm{OH}\right)_{2}\left[\mathrm{~S}_{6} \mathrm{P}_{2}\left\{\mathrm{OCH}_{2} \mathrm{C}\left(\mathrm{CH}_{3}\right)_{2} \mathrm{CH}_{2} \mathrm{O}\right\}_{2}\right]$ & $\begin{array}{c}1030 \\
(1027.8)\end{array}$ & $\begin{array}{c}9.28 \\
(9.32)\end{array}$ & $\begin{array}{c}18.62 \\
(18.68)\end{array}$ & $\begin{array}{c}46.63 \\
(46.70)\end{array}$ & $\begin{array}{c}5.39 \\
(5.45)\end{array}$ & $\begin{array}{c}4.52 \\
(4.48)\end{array}$ & - \\
\hline 5 & 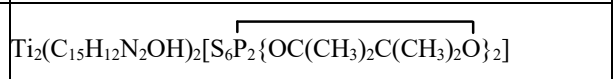 & $\begin{array}{c}1055 \\
(1055.8)\end{array}$ & $\begin{array}{c}9.01 \\
(9.07)\end{array}$ & $\begin{array}{c}18.23 \\
(18.19)\end{array}$ & $\begin{array}{c}47.70 \\
(47.74)\end{array}$ & $\begin{array}{c}5.28 \\
(5.30)\end{array}$ & $\begin{array}{c}4.80 \\
(4.74)\end{array}$ & - \\
\hline 6 & 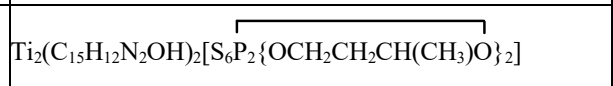 & $\begin{array}{c}995 \\
(996.8)\end{array}$ & $\begin{array}{c}9.54 \\
(9.61)\end{array}$ & $\begin{array}{c}19.20 \\
(19.26)\end{array}$ & $\begin{array}{c}45.81 \\
(45.75)\end{array}$ & $\begin{array}{c}5.66 \\
(5.61)\end{array}$ & $\begin{array}{c}3.45 \\
(3.41)\end{array}$ & - \\
\hline 7 & $\mathrm{Ti}_{2}\left(\mathrm{C}_{15} \mathrm{H}_{12} \mathrm{~N}_{2} \mathrm{OCH}_{3}\right)_{2}\left[\mathrm{~S}_{6} \mathrm{P}_{2}\left(\mathrm{OCH}_{2} \mathrm{CH}_{2} \mathrm{CH}_{3}\right)_{4}\right]$ & $\begin{array}{c}1085 \\
(1087.8)\end{array}$ & $\begin{array}{c}8.78 \\
(8.81)\end{array}$ & $\begin{array}{c}17.60 \\
(17.65)\end{array}$ & $\begin{array}{c}48.61 \\
(48.54)\end{array}$ & $\begin{array}{c}5.23 \\
(5.15)\end{array}$ & $\begin{array}{c}3.82 \\
(3.86)\end{array}$ & - \\
\hline 8 & $\mathrm{Ti}_{2}\left(\mathrm{C}_{15} \mathrm{H}_{12} \mathrm{~N}_{2} \mathrm{OCH}_{3}\right)_{2}\left[\mathrm{~S}_{6} \mathrm{P}_{2}\left(\mathrm{OC}_{6} \mathrm{H}_{5}\right)_{4}\right]$ & $\begin{array}{c}1125 \\
(1223.8)\end{array}$ & $\begin{array}{c}7.80 \\
(7.83)\end{array}$ & $\begin{array}{c}18.74 \\
(18.69)\end{array}$ & $\begin{array}{c}54.85 \\
(54.91)\end{array}$ & $\begin{array}{c}4.52 \\
(4.58)\end{array}$ & $\begin{array}{c}4.02 \\
(4.09)\end{array}$ & - \\
\hline 9 & 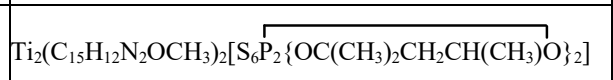 & $\begin{array}{c}1080 \\
(1083.8)\end{array}$ & $\begin{array}{l}8.80 \\
(8.84\end{array}$ & $\begin{array}{r}17.85 \\
(17.72\end{array}$ & $\begin{array}{l}48.79 \\
(48.72\end{array}$ & $\begin{array}{l}5.13 \\
(5.17\end{array}$ & $\begin{array}{l}4.92 \\
(4.98\end{array}$ & - \\
\hline 10 & $\mathrm{Ti}_{2}\left(\mathrm{C}_{15} \mathrm{H}_{12} \mathrm{~N}_{2} \mathrm{OCH}_{3}\right)_{2}\left[\mathrm{~S}_{6} \mathrm{P}_{2}\left\{\mathrm{OCH}_{2} \mathrm{C}\left(\mathrm{CH}_{3}\right)_{2} \mathrm{CH}_{2} \mathrm{O}\right\}_{2}\right]$ & $\begin{array}{c}1055 \\
(1055.8)\end{array}$ & $\begin{array}{c}9.15 \\
(9.07)\end{array}$ & $\begin{array}{c}18.16 \\
(18.19)\end{array}$ & $\begin{array}{c}47.80 \\
(47.74)\end{array}$ & $\begin{array}{c}5.25 \\
(5.30)\end{array}$ & $\begin{array}{c}4.70 \\
(4.74)\end{array}$ & - \\
\hline 11 & 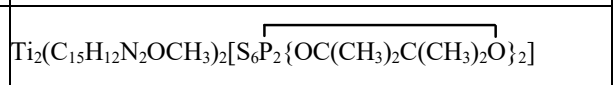 & $\begin{array}{c}1082 \\
(1083.8)\end{array}$ & $\begin{array}{c}8.80 \\
(8.84)\end{array}$ & $\begin{array}{c}17.68 \\
(17.72)\end{array}$ & $\begin{array}{c}48.78 \\
(48.72)\end{array}$ & $\begin{array}{c}5.23 \\
(5.17)\end{array}$ & $\begin{array}{c}5.02 \\
(4.98)\end{array}$ & - \\
\hline 12 & 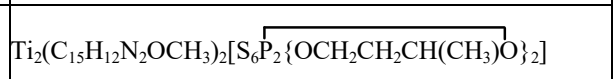 & $\begin{array}{c}1025 \\
(1024.8)\end{array}$ & $\begin{array}{c}9.28 \\
(9.34)\end{array}$ & $\begin{array}{c}18.65 \\
(18.74)\end{array}$ & $\begin{array}{c}46.78 \\
(46.84)\end{array}$ & $\begin{array}{c}5.42 \\
(5.46)\end{array}$ & $\begin{array}{c}4.42 \\
(4.49)\end{array}$ & - \\
\hline 13 & $\mathrm{Ti}_{2}\left(\mathrm{C}_{15} \mathrm{H}_{12} \mathrm{~N}_{2} \mathrm{O}_{2} \mathrm{CH}_{3}\right)_{2}\left[\mathrm{~S}_{6} \mathrm{P}_{2}\left(\mathrm{OCH}_{2} \mathrm{CH}_{2} \mathrm{CH}_{3}\right)_{4}\right]$ & $\begin{array}{c}1120 \\
(1119.8)\end{array}$ & $\begin{array}{c}8.48 \\
(8.56)\end{array}$ & $\begin{array}{c}17.10 \\
(17.15)\end{array}$ & $\begin{array}{c}47.21 \\
(47.15)\end{array}$ & $\begin{array}{c}5.07 \\
(5.00)\end{array}$ & $\begin{array}{c}5.12 \\
(5.18)\end{array}$ & - \\
\hline 14 & $\mathrm{Ti}_{2}\left(\mathrm{C}_{15} \mathrm{H}_{12} \mathrm{~N}_{2} \mathrm{O}_{2} \mathrm{CH}_{3}\right)_{2}\left[\mathrm{~S}_{6} \mathrm{P}_{2}\left(\mathrm{OC}_{6} \mathrm{H}_{5}\right)_{4}\right]$ & $\begin{array}{c}1260 \\
(1255.8)\end{array}$ & $\begin{array}{c}7.60 \\
(7.63)\end{array}$ & $\begin{array}{c}15.22 \\
(15.29)\end{array}$ & $\begin{array}{c}53.42 \\
(53.51)\end{array}$ & $\begin{array}{c}4.51 \\
(4.46)\end{array}$ & $\begin{array}{c}3.96 \\
(3.98)\end{array}$ & - \\
\hline 15 & $\mathrm{Ti}_{2}\left(\mathrm{C}_{15} \mathrm{H}_{12} \mathrm{~N}_{2} \mathrm{O}_{2} \mathrm{CH}_{3}\right)_{2}\left[\mathrm{~S}_{6} \mathrm{P}_{2}\left\{\mathrm{OC}\left(\mathrm{CH}_{3}\right)_{2} \mathrm{CH}_{2} \mathrm{CH}\left(\mathrm{CH}_{3}\right) \mathrm{O}\right\}_{2}\right]$ & $\begin{array}{c}1160 \\
(1161.8)\end{array}$ & $\begin{array}{c}8.31 \\
(8.25)\end{array}$ & $\begin{array}{c}16.50 \\
(16.53)\end{array}$ & $\begin{array}{c}45.52 \\
(45.45)\end{array}$ & $\begin{array}{c}4.87 \\
(4.82)\end{array}$ & $\begin{array}{c}4.68 \\
(4.64)\end{array}$ & - \\
\hline 16 & $\mathrm{Ti}_{2}\left(\mathrm{C}_{15} \mathrm{H}_{12} \mathrm{~N}_{2} \mathrm{O}_{2} \mathrm{CH}_{3}\right)_{2}\left[\mathrm{~S}_{6} \mathrm{P}_{2}\left\{\mathrm{OCH}_{2} \mathrm{C}\left(\mathrm{CH}_{3}\right)_{2} \mathrm{CH}_{2} \mathrm{O}\right\}_{2}\right]$ & $\begin{array}{c}1045 \\
(1042.8)\end{array}$ & $\begin{array}{c}9.17 \\
(9.19)\end{array}$ & $\begin{array}{c}18.35 \\
(18.41)\end{array}$ & $\begin{array}{c}48.28 \\
(48.33)\end{array}$ & $\begin{array}{c}5.42 \\
(5.37)\end{array}$ & $\begin{array}{c}4.75 \\
(4.79)\end{array}$ & - \\
\hline 17 & $\mathrm{Ti}_{2}\left(\mathrm{C}_{15} \mathrm{H}_{12} \mathrm{~N}_{2} \mathrm{O}_{2} \mathrm{CH}_{3}\right)_{2}\left[\mathrm{~S}_{6} \mathrm{P}_{2}\left\{\mathrm{OC}\left(\mathrm{CH}_{3}\right)_{2} \mathrm{C}\left(\mathrm{CH}_{3}\right)_{2} \mathrm{O}\right\}_{2}\right]$ & $\begin{array}{c}1160 \\
(1161.8)\end{array}$ & $\begin{array}{c}8.19 \\
(8.25)\end{array}$ & $\begin{array}{c}16.50 \\
(16.53)\end{array}$ & $\begin{array}{c}43.29 \\
(43.38)\end{array}$ & $\begin{array}{l}4.78 \\
(4.82)\end{array}$ & $\begin{array}{c}4.60 \\
(4.64)\end{array}$ & - \\
\hline 18 & $\mathrm{Ti}_{2}\left(\mathrm{C}_{15} \mathrm{H}_{12} \mathrm{~N}_{2} \mathrm{O}_{2} \mathrm{CH}_{3}\right)_{2}\left[\mathrm{~S}_{6} \mathrm{P}_{2}\left\{\mathrm{OCH}_{2} \mathrm{CH}_{2} \mathrm{CH}\left(\mathrm{CH}_{3}\right) \mathrm{O}_{2}\right]\right.$ & $\begin{array}{c}1015 \\
(1011.8)\end{array}$ & $\begin{array}{l}9.36 \\
(9.47)\end{array}$ & $\begin{array}{c}18.91 \\
(18.98)\end{array}$ & $\begin{array}{c}47.40 \\
(47.44)\end{array}$ & $\begin{array}{c}5.50 \\
(5.53)\end{array}$ & $\begin{array}{c}4.50 \\
(4.55)\end{array}$ & - \\
\hline
\end{tabular}

Bull. Chem. Soc. Ethiop. 2021, 35(1) 


\begin{tabular}{|c|c|c|c|c|c|c|c|c|}
\hline 19 & $\mathrm{Ti}_{2}\left(\mathrm{C}_{15} \mathrm{H}_{12} \mathrm{~N}_{2} \mathrm{OCl}\right)_{2}\left[\mathrm{~S}_{6} \mathrm{P}_{2}\left(\mathrm{OCH}_{2} \mathrm{CH}_{2} \mathrm{CH}_{3}\right)_{4}\right]$ & $\begin{array}{c}1130 \\
(1128.8)\end{array}$ & $\begin{array}{l}8.441 \\
(8.49)\end{array}$ & $\begin{array}{c}16.99 \\
(17.00)\end{array}$ & $\begin{array}{c}46.65 \\
(46.78)\end{array}$ & $\begin{array}{c}4.93 \\
(4.96)\end{array}$ & $\begin{array}{c}4.63 \\
(4.61)\end{array}$ & $\begin{array}{c}6.25 \\
(6.28)\end{array}$ \\
\hline 20 & $\mathrm{Ti}_{2}\left(\mathrm{C}_{15} \mathrm{H}_{12} \mathrm{~N}_{2} \mathrm{OCl}\right)_{2}\left[\mathrm{~S}_{6} \mathrm{P}_{2}\left(\mathrm{OC}_{6} \mathrm{H}_{5}\right)_{4}\right]$ & $\begin{array}{c}1265 \\
(1264.8)\end{array}$ & $\begin{array}{c}7.69 \\
(7.57)\end{array}$ & $\begin{array}{c}15.13 \\
(15.18)\end{array}$ & $\begin{array}{c}51.17 \\
(51.29)\end{array}$ & $\begin{array}{r}4.52 \\
(4.49)\end{array}$ & $\begin{array}{c}3.46 \\
(3.48)\end{array}$ & $\begin{array}{c}5.64 \\
(5.61)\end{array}$ \\
\hline 21 & $\mathrm{Ti}_{2}\left(\mathrm{C}_{15} \mathrm{H}_{12} \mathrm{~N}_{2} \mathrm{OCl}\right)_{2}\left[\mathrm{~S}_{6} \stackrel{\mathrm{P}_{2}\left\{\mathrm{OC}\left(\mathrm{CH}_{3}\right)_{2} \mathrm{CH}_{2} \mathrm{CH}\left(\mathrm{CH}_{3}\right) \mathrm{O}\right.}{\mathrm{C}_{2}}\right]$ & $\begin{array}{c}1120 \\
(1124.8)\end{array}$ & $\begin{array}{c}8.47 \\
(8.52)\end{array}$ & $\begin{array}{c}17.17 \\
(17.07)\end{array}$ & $\begin{array}{l}44.90 \\
(44.81)\end{array}$ & $\begin{array}{c}4.91 \\
(4.98)\end{array}$ & $\begin{array}{c}4.23 \\
(4.27)\end{array}$ & $\begin{array}{r}6.29 \\
(6.31)\end{array}$ \\
\hline 22 & $\mathrm{Ti}_{2}\left(\mathrm{C}_{15} \mathrm{H}_{12} \mathrm{~N}_{2} \mathrm{OCl}\right)_{2}\left[\mathrm{~S}_{6} \mathrm{P}_{2}\left\{\mathrm{OCH}_{2} \mathrm{C}\left(\mathrm{CH}_{3}\right)_{2} \mathrm{CH}_{2} \mathrm{O}\right\}_{2}\right]$ & $\begin{array}{c}1095 \\
(1096.8)\end{array}$ & $\begin{array}{c}8.68 \\
(8.73)\end{array}$ & $\begin{array}{r}17.45 \\
(17.51)\end{array}$ & $\begin{array}{l}43.85 \\
(43.76)\end{array}$ & $\begin{array}{c}5.04 \\
(5.11)\end{array}$ & $\begin{array}{c}4.05 \\
(4.01)\end{array}$ & $\begin{array}{r}6.50 \\
(6.47)\end{array}$ \\
\hline 23 & $\mathrm{Ti}_{2}\left(\mathrm{C}_{15} \mathrm{H}_{12} \mathrm{~N}_{2} \mathrm{OCl}\right)_{2}\left[\mathrm{~S}_{6} \mathrm{P}_{2}\left\{\mathrm{OC}\left(\mathrm{CH}_{3}\right)_{2} \mathrm{C}\left(\mathrm{CH}_{3}\right)_{2} \mathrm{O}\right\}_{2}\right]$ & $\begin{array}{c}1125 \\
(1124.8)\end{array}$ & $\begin{array}{l}8.49 \\
(8.52\end{array}$ & $\begin{array}{c}17.15 \\
(17.07)\end{array}$ & $\begin{array}{c}44.69 \\
(44.81)\end{array}$ & $\begin{array}{r}4.93 \\
(4.98)\end{array}$ & $\begin{array}{c}4.24 \\
(4.27)\end{array}$ & $\begin{array}{r}6.29 \\
(6.31)\end{array}$ \\
\hline 24 & 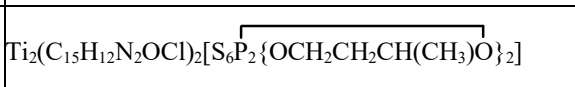 & $\begin{array}{c}1065 \\
(1065.8)\end{array}$ & $\begin{array}{c}8.91 \\
(8.99)\end{array}$ & $\begin{array}{c}18.07 \\
(18.01)\end{array}$ & $\begin{array}{l}42.88 \\
(42.78)\end{array}$ & $\begin{array}{c}5.19 \\
(5.25)\end{array}$ & $\begin{array}{c}3.76 \\
(3.75)\end{array}$ & $\begin{array}{c}6.62 \\
(6.66)\end{array}$ \\
\hline
\end{tabular}

\section{${ }^{I} H$ NMR spectra analysis}

The ${ }^{1} \mathrm{H}$ NMR spectra of synthesized mixed ligand complexes, recorded in $\mathrm{CDCl}_{3}$ exhibit characteristic signals (Table 3 ). In the region $\delta 7.42-6.39 \mathrm{ppm}$, a very complex pattern may be assigned to the aromatic protons of ligand pyrazoline [47]. The pyrazoline ligand exhibits a characteristic peak at $\delta \sim 11.00 \mathrm{ppm}$ due to hydroxyl protons, the absence of that particular peak in the ${ }^{1} \mathrm{H}$ NMR spectra of the metal complex suggests that the hydroxyl oxygen atom is bonded to Ti metal. A broad singlet peak observed at $\delta 5.37-4.86 \mathrm{ppm}$ may be attributed to the N-H group (primarily at $\delta 5.40-4.90 \mathrm{ppm}$ in free pyrazoline) indicating that the $-\mathrm{NH}$ group is not involved in metal complex formation [47]. The bands at 3.82-3.07 and 2.25-2.02 ppm could be ascribed, respectively to $-\mathrm{CH}$ and $-\mathrm{CH}_{2}$ groups. The band at $\delta 5.54-4.19 \mathrm{ppm}$ for $-\mathrm{OCH}_{2}$ and at $\delta$ 4.94-4.21 ppm for -OCH group and bands for methyl group are observed at $\delta 1.10-0.90 \mathrm{ppm}$. The complex pattern observed at $\delta 7.21-7.04 \mathrm{ppm}$ may be due to the skeletal protons of the phenyl ring. The hydrogen atom calculated through the integrations ratio suggests that two of the dithiophosphate ligands and two pyrazoline ligands are present in synthesized mixed ligand complexes.

${ }^{31} P$ NMR spectra analysis

The synthesized compounds exhibit only one signal for the phosphorus atoms in protondecoupled ${ }^{31} \mathrm{P}$ NMR spectra. The ${ }^{31} \mathrm{P}$ NMR signals of Ti dichlorodithio-compounds are obtained at $\delta=90.0 \mathrm{ppm}$ while that of synthesized Ti mixed ligand complexes are observed at $\delta=110.0$ $91.3 \mathrm{ppm}$. The downfield shifting of the signal due to dithiophosphato phosphorus atom at about $\delta 15.0 \mathrm{ppm}$ confirms the bidentate nature of the ligand [48]. Although two phosphorus atoms are there only one signal is obtained, indicates a similar environment for both the phosphorus atom (Table 3). 
Table 2. IR spectral data $\left(\mathrm{cm}^{-1}\right)$ for $\mathrm{Ti}_{2}\left(\mathrm{C}_{15} \mathrm{H}_{12} \mathrm{~N}_{2} \mathrm{OH}\right)_{2}\left[\mathrm{~S}_{6} \mathrm{P}_{2}(\mathrm{OR})_{4}\right]$.

\begin{tabular}{|c|c|c|c|c|c|c|c|c|c|c|c|}
\hline S. No. & $v[\mathrm{~N}-\mathrm{H}]$ & $v[\mathrm{C}=\mathrm{N}]$ & $v[\mathrm{C}-\mathrm{O}]$ & $v[(\mathrm{P})-\mathrm{O}-\mathrm{C}]$ & $v[\mathrm{P}-\mathrm{O}-(\mathrm{C})]$ & $v[\mathrm{P}=\mathrm{S}]$ & $v[\mathrm{P}-\mathrm{S}]$ & Ring vib & $v[\mathrm{Ti}-\mathrm{O}]$ & $v[\mathrm{Ti}-\mathrm{S}]$ & $v[\mathrm{Ti}-\mathrm{N}]$ \\
\hline 1 & 3329 & 1620 & - & 1058 & 842 & 654 & 546 & -- & 463 & $\begin{array}{c}335 \\
295 \mathrm{~S}_{\mathrm{b}} \\
\end{array}$ & 387 \\
\hline 2 & 3339 & 1616 & - & 1075 & 846 & 650 & 537 & - & 471 & $\begin{array}{c}327 \\
290 \mathrm{~S}_{\mathrm{b}} \\
\end{array}$ & 391 \\
\hline 3 & 3341 & 1604 & - & 1036 & 878 & 692 & 529 & 961 & 485 & $\begin{array}{c}330 \\
294 S_{b}\end{array}$ & 396 \\
\hline 4 & 3328 & 1619 & - & 1067 & 842 & 647 & 549 & 957 & 467 & $\begin{array}{c}325 \\
302 \mathrm{~S}_{\mathrm{b}}\end{array}$ & 389 \\
\hline 5 & 3329 & 1623 & - & 1068 & 832 & 662 & 538 & 948 & 468 & $\begin{array}{c}327 \\
300 \mathrm{~S}_{\mathrm{b}}\end{array}$ & 387 \\
\hline 6 & 3339 & 1621 & - & 1069 & 832 & 656 & 548 & 978 & 460 & $\begin{array}{c}321 \\
298 \mathrm{~S}_{\mathrm{b}}\end{array}$ & 381 \\
\hline 7 & 3336 & 1614 & - & 1073 & 832 & 646 & 529 & - & 463 & $\begin{array}{c}326 \\
295 \mathrm{~S}_{\mathrm{b}} \\
\end{array}$ & 384 \\
\hline 8 & 3328 & 1618 & - & 1052 & 898 & 658 & 546 & - & 471 & $\begin{array}{c}321 \\
297 \mathrm{~S}_{\mathrm{b}}\end{array}$ & 393 \\
\hline 9 & 3328 & 1619 & - & 1073 & 833 & 649 & 537 & 968 & 470 & $\begin{array}{c}325 \\
298 \mathrm{~S}_{\mathrm{b}}\end{array}$ & 394 \\
\hline 10 & 3340 & 1620 & - & 1067 & 824 & 664 & 546 & 947 & 472 & $\begin{array}{c}329 \\
302 \mathrm{~S}_{\mathrm{b}}\end{array}$ & 387 \\
\hline 11 & 3328 & 1612 & - & 1078 & 835 & 648 & 529 & 958 & 463 & $\begin{array}{c}328 \\
297 \mathrm{~S}_{\mathrm{b}}\end{array}$ & 390 \\
\hline 12 & 3342 & 1617 & - & 1058 & 836 & 648 & 547 & 973 & 467 & $\begin{array}{c}331 \\
298 \mathrm{~S}_{\mathrm{b}}\end{array}$ & 395 \\
\hline 13 & 3329 & 1621 & 1025 & 1059 & 892 & 657 & 533 & - & 466 & $\begin{array}{c}335 \\
301 \mathrm{~S}_{\mathrm{b}}\end{array}$ & 383 \\
\hline 14 & 3327 & 1623 & 1022 & 1065 & 872 & 669 & 539 & - & 469 & $\begin{array}{c}322 \\
294 S_{b}\end{array}$ & 382 \\
\hline 15 & 3338 & 1618 & 1015 & 1076 & 835 & 650 & 546 & 940 & 470 & $\begin{array}{c}321 \\
301 \mathrm{~S}_{\mathrm{b}}\end{array}$ & 389 \\
\hline 16 & 3325 & 1619 & 1030 & 1062 & 894 & 649 & 529 & 965 & 474 & $\begin{array}{c}324 \\
300 \mathrm{~S}_{\mathrm{b}} \\
\end{array}$ & 393 \\
\hline 17 & 3331 & 1623 & 1023 & 1064 & 898 & 673 & 537 & 948 & 468 & $\begin{array}{c}331 \\
298 \mathrm{~S}_{\mathrm{b}}\end{array}$ & 391 \\
\hline 18 & 3327 & 1614 & 1017 & 1076 & 826 & 659 & 546 & 965 & 463 & $\begin{array}{c}329 \\
295 \mathrm{~S}_{\mathrm{b}} \\
\end{array}$ & 381 \\
\hline 19 & 3345 & 1618 & - & 1050 & 835 & 663 & 528 & - & 469 & $\begin{array}{c}321 \\
299 \mathrm{~S}_{\mathrm{b}}\end{array}$ & 388 \\
\hline 20 & 3343 & 1619 & - & 1074 & 843 & 653 & 537 & - & 466 & $\begin{array}{c}326 \\
301 \mathrm{~S}_{\mathrm{b}} \\
\end{array}$ & 380 \\
\hline 21 & 3329 & 1620 & - & 1058 & 834 & 659 & 530 & 955 & 465 & $\begin{array}{c}322 \\
294 \mathrm{~S}_{\mathrm{b}}\end{array}$ & 394 \\
\hline 22 & 3330 & 1612 & - & 1072 & 826 & 660 & 531 & 953 & 461 & $\begin{array}{c}324 \\
298 \mathrm{~S}_{\mathrm{b}} \\
\end{array}$ & 387 \\
\hline 23 & 3340 & 1623 & - & 1057 & 899 & 670 & 537 & 961 & 474 & $\begin{array}{c}329 \\
293 S_{b} \\
\end{array}$ & 386 \\
\hline 24 & 3346 & 1622 & - & 1078 & 887 & 658 & 542 & 970 & 475 & $\begin{array}{c}320 \\
302 \mathrm{~S}_{\mathrm{b}}\end{array}$ & 383 \\
\hline
\end{tabular}




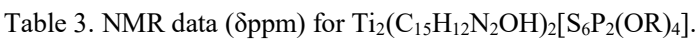

\begin{tabular}{|c|c|c|c|c|}
\hline \multirow{2}{*}{ S. No. } & \multirow{2}{*}{ Compound } & \multicolumn{3}{|c|}{ Chemical shift $(\delta \mathrm{ppm})$} \\
\hline & & ${ }^{1} \mathrm{H}$ NMR & ${ }^{13} \mathrm{C}$ NMR & ${ }^{31} \mathrm{P}$ NMR \\
\hline 1 & $\mathrm{Ti}_{2}\left(\mathrm{C}_{15} \mathrm{H}_{12} \mathrm{~N}_{2} \mathrm{OH}\right)_{2}\left[\mathrm{~S}_{6} \mathrm{P}_{2}\left(\mathrm{OCH}_{2} \mathrm{CH}_{2} \mathrm{CH}_{3}\right)_{4}\right]$ & $\begin{array}{l}7.65-6.80(\mathrm{~m}, 18 \mathrm{H}, \\
\mathrm{Ar}-\mathrm{H}) \\
5.12\left(\mathrm{~m}, 8 \mathrm{H},-\mathrm{OCH}_{2}-\right) \\
0.92\left(\mathrm{t}, 12 \mathrm{H},-\mathrm{CH}_{3}\right) \\
1.68\left(\mathrm{~m}, 8 \mathrm{H},-\mathrm{CH}_{2}\right) \\
5.18(\mathrm{~s}, 2 \mathrm{H}, \mathrm{NH}) \\
3.32(\mathrm{t}, 2 \mathrm{H},-\mathrm{CH}) \\
2.15\left(\mathrm{~d}, 4 \mathrm{H},-\mathrm{CH}_{2}\right)\end{array}$ & - & 106.10 \\
\hline 2 & $\mathrm{Ti}_{2}\left(\mathrm{C}_{15} \mathrm{H}_{12} \mathrm{~N}_{2} \mathrm{OH}\right)_{2}\left[\mathrm{~S}_{6} \mathrm{P}_{2}\left(\mathrm{OC}_{6} \mathrm{H}_{5}\right)_{4}\right]$ & $\begin{array}{l}\text { 7.47-6.89 (m, } 18 \mathrm{H}, \\
\mathrm{Ar}-\mathrm{H}) \\
7.14\left(\mathrm{~s}, 20 \mathrm{H},-\mathrm{C}_{6} \mathrm{H}_{5}\right) \\
4.87(\mathrm{~s}, 2 \mathrm{H},-\mathrm{NH}) \\
3.18(\mathrm{t}, 4 \mathrm{H},-\mathrm{CH}) \\
2.49\left(\mathrm{~d}, 8 \mathrm{H},-\mathrm{CH}_{2}\right)\end{array}$ & - & 92.40 \\
\hline 3 & $\left.\mathrm{Ti}_{2}\left(\mathrm{C}_{15} \mathrm{H}_{12} \mathrm{~N}_{2} \mathrm{OH}\right)_{2}[\mathrm{~S}_{6} \overbrace{\mathrm{P}_{2}\left\{\mathrm{OC}\left(\mathrm{CH}_{3}\right)_{2} \mathrm{CH}_{2} \mathrm{CH}\left(\mathrm{CH}_{3}\right) \mathrm{O}\right.}\}_{2}\right]$ & \begin{tabular}{|l|}
$7.59-6.85(\mathrm{~m}, 18 \mathrm{H}$, \\
$\mathrm{Ar}-\mathrm{H})$ \\
$2.97-2.60\left(\mathrm{~m}, 22 \mathrm{H},-\mathrm{CH}_{3}\right.$, \\
$\left.-\mathrm{CH}_{2}\right)$ \\
$4.19(\mathrm{~m}, 2 \mathrm{H},-\mathrm{OCH})$ \\
$5.14(\mathrm{~s}, 24 \mathrm{H},-\mathrm{NH})$ \\
$3.52(\mathrm{t}, 2 \mathrm{H},-\mathrm{CH})$ \\
$2.18\left(\mathrm{~d}, 8 \mathrm{H},-\mathrm{CH}_{2}\right)$ \\
\end{tabular} & \begin{tabular}{|l}
$91.52(-\mathrm{OC}, \mathrm{dtp})$ \\
$24.64\left(-\mathrm{CH}_{3}, \mathrm{dtp}\right)$ \\
$76.93(-\mathrm{OCH}, \mathrm{dtp})$ \\
$26.46\left(-\mathrm{CH}_{2}, \mathrm{dtp}\right)$ \\
$136.82(\mathrm{Ar}-\mathrm{C})$ \\
$167.43(\mathrm{C}=\mathrm{N})$ \\
$43.05(-\mathrm{CH})$ \\
$25.97\left(-\mathrm{CH}_{2}\right)$ \\
\end{tabular} & 93.60 \\
\hline 4 & $\mathrm{Ti}_{2}\left(\mathrm{C}_{15} \mathrm{H}_{12} \mathrm{~N}_{2} \mathrm{OH}\right)_{2}\left[\mathrm{~S}_{6} \mathrm{P}_{2}\left\{\mathrm{OCH}_{2} \mathrm{C}\left(\mathrm{CH}_{3}\right)_{2} \mathrm{CH}_{2} \mathrm{O}\right\}_{2}\right]$ & $\begin{array}{l}7.64-6.83(\mathrm{~m}, 18 \mathrm{H}, \\
\mathrm{Ar}-\mathrm{H}) \\
0.98\left(\mathrm{~s}, 12 \mathrm{H},-\mathrm{CH}_{3}\right) \\
4.24\left(\mathrm{~d}, 8 \mathrm{H},-\mathrm{OCH}_{2}-\right) \\
4.84(\mathrm{~s}, 4 \mathrm{H}, \mathrm{NH}) \\
3.03(\mathrm{t}, 2 \mathrm{H},-\mathrm{CH}) \\
2.25\left(\mathrm{~d}, 8 \mathrm{H},-\mathrm{CH}_{2}\right)\end{array}$ & $\begin{array}{l}21.95\left(\mathrm{CH}_{3}, \mathrm{dtp}\right) \\
32.72(\mathrm{q} \mathrm{C}, \mathrm{dtp}) \\
76.02 \quad\left(\mathrm{~d}, \quad-\mathrm{OCH}_{2},\right. \\
\mathrm{dtp}) \\
136.74(\mathrm{Ar}-\mathrm{C}) \\
27.63\left(-\mathrm{CH}_{2}\right) \\
42.31(-\mathrm{CH}) \\
165.56(\mathrm{C}=\mathrm{N})\end{array}$ & 91.50 \\
\hline 5 & $\mathrm{Ti}_{2}\left(\mathrm{C}_{15} \mathrm{H}_{12} \mathrm{~N}_{2} \mathrm{OH}\right)_{2}\left[\mathrm{~S}_{6}{\stackrel{\mathrm{P}}{2}\left[\mathrm{OC}\left(\mathrm{CH}_{3}\right)_{2} \mathrm{C}\left(\mathrm{CH}_{3}\right)_{2} \mathrm{O}\right.}_{\}_{2}}\right]$ & $\begin{array}{l}1.02\left(\mathrm{~s}, 24 \mathrm{H},-\mathrm{CH}_{3}\right) \\
7.69-6.82(\mathrm{~m}, 18 \mathrm{H}, \mathrm{ArH}) \\
4.89(\mathrm{~s}, 4 \mathrm{H},-\mathrm{NH}) \\
3.15(\mathrm{t}, 2 \mathrm{H},-\mathrm{CH}) \\
2.25\left(\mathrm{~d}, 4 \mathrm{H},-\mathrm{CH}_{2}\right)\end{array}$ & $\begin{array}{l}24.45\left(-\mathrm{CH}_{3}, \mathrm{dtp}\right) \\
91.92(-\mathrm{OC}, \mathrm{dtp}) \\
27.42\left(-\mathrm{CH}_{2}\right) \\
136.81-123.12 \\
(\mathrm{Ar}-\mathrm{C}) \\
43.08(-\mathrm{CH}) \\
163.65(\mathrm{C}=\mathrm{N})\end{array}$ & 104.82 \\
\hline 6 & 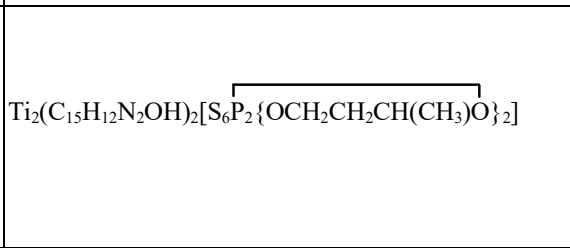 & \begin{tabular}{|l|}
$4.12-3.35(\mathrm{~m}, 6 \mathrm{H}$, \\
$\left.-\mathrm{OCH}_{2}, \mathrm{OCH}\right)$ \\
$2.65-1.09\left(\mathrm{~m}, 10 \mathrm{H},-\mathrm{CH}_{3}\right.$, \\
$\left.\mathrm{CH}_{2}\right)$ \\
$7.68-6.42(\mathrm{~m}, \mathrm{ArH})$ \\
$5.16(\mathrm{~s}, 4 \mathrm{H},-\mathrm{NH})$ \\
$3.07(\mathrm{t}, 2 \mathrm{H},-\mathrm{CH})$ \\
$2.17\left(\mathrm{~d}, 8 \mathrm{H},-\mathrm{CH}_{2}\right)$
\end{tabular} & $\begin{array}{l}23.95\left(-\mathrm{CH}_{3}, \mathrm{dtp}\right) \\
76.34(-\mathrm{OCH}, \\
\left.-\mathrm{OCH}_{2,} \mathrm{dtp}\right) \\
27.72\left(-\mathrm{CH}_{2}\right) \\
135.57-123.19 \\
(\mathrm{Ar}-\mathrm{C}) \\
43.19(-\mathrm{CH}) \\
162.94(\mathrm{C}=\mathrm{N})\end{array}$ & 107.00 \\
\hline 7 & $\mathrm{Ti}_{2}\left(\mathrm{C}_{15} \mathrm{H}_{12} \mathrm{~N}_{2} \mathrm{OCH}_{3}\right)_{2}\left[\mathrm{~S}_{6} \mathrm{P}_{2}\left(\mathrm{OCH}_{2} \mathrm{CH}_{2} \mathrm{CH}_{3}\right)_{4}\right]$ & $\begin{array}{l}\text { 7.63-6.65 (m, 16H, } \\
\text { Ar-H) } \\
1.34\left(\mathrm{~m}, 8 \mathrm{H},-\mathrm{CH}_{2}\right) \\
4.25\left(\mathrm{~m}, 8 \mathrm{H},-\mathrm{OCH}_{2}-\right) \\
0.96\left(\mathrm{t}, 12 \mathrm{H},-\mathrm{CH}_{3}\right) \\
5.10(\mathrm{~s}, 2 \mathrm{H}, \mathrm{NH}) \\
3.42(\mathrm{t}, 2 \mathrm{H},-\mathrm{CH}) \\
2.10\left(\mathrm{~d}, 4 \mathrm{H},-\mathrm{CH}_{2}\right) \\
0.97\left(\mathrm{~s}, 6 \mathrm{H},-\mathrm{CH}_{3}\right)\end{array}$ & - & 105.82 \\
\hline 8 & & $7.61-6.87(\mathrm{~m}, 16 \mathrm{H}$ & - & 92.72 \\
\hline
\end{tabular}

Bull. Chem. Soc. Ethiop. 2021, 35(1) 


\begin{tabular}{|c|c|c|c|c|}
\hline & $\mathrm{Ti}_{2}\left(\mathrm{C}_{15} \mathrm{H}_{12} \mathrm{~N}_{2} \mathrm{OCH}_{3}\right)_{2}\left[\mathrm{~S}_{6} \mathrm{P}_{2}\left(\mathrm{OC}_{6} \mathrm{H}_{5}\right)_{4}\right]$ & \begin{tabular}{|l|}
$\mathrm{Ar}-\mathrm{H})$ \\
$7.21\left(\mathrm{~s}, 2 \mathrm{H},-\mathrm{C}_{6} \mathrm{H}_{5}\right)$ \\
$5.12(\mathrm{~s}, 2 \mathrm{H},-\mathrm{NH})$ \\
$3.26(\mathrm{t}, 4 \mathrm{H},-\mathrm{CH})$ \\
$2.17\left(\mathrm{~d}, 8 \mathrm{H}-\mathrm{CH}_{2}\right)$ \\
\end{tabular} & & \\
\hline 9 & $\mathrm{Ti}_{2}\left(\mathrm{C}_{15} \mathrm{H}_{12} \mathrm{~N}_{2} \mathrm{OCH}_{3}\right)_{2}\left[\mathrm{~S}_{6} \mathrm{P}_{2}\left\{\mathrm{OC}\left(\mathrm{CH}_{3}\right)_{2} \mathrm{CH}_{2} \mathrm{CH}\left(\mathrm{CH}_{3}\right) \mathrm{O}\right\}_{2}\right]$ & $\begin{array}{l}7.52-6.72(\mathrm{~m}, 16 \mathrm{H}, \\
\mathrm{Ar}-\mathrm{H}) \\
2.56-2.09\left(\mathrm{~m}, 22 \mathrm{H},-\mathrm{CH}_{3},\right. \\
\left.\mathrm{CH}_{2}\right) \\
4.94-4.58(\mathrm{~m}, 2 \mathrm{H},-\mathrm{OCH}) \\
5.15(\mathrm{~s}, 4 \mathrm{H},-\mathrm{NH}) \\
3.27(\mathrm{t}, 2 \mathrm{H},-\mathrm{CH}) \\
2.12\left(\mathrm{~d}, 8 \mathrm{H}, \mathrm{CH}_{2}\right) \\
0.96\left(\mathrm{~s}, 6 \mathrm{H},-\mathrm{CH}_{3}\right)\end{array}$ & \begin{tabular}{|l}
91.23 (-OC, dtp) \\
25.91 (-CH, dtp) \\
76.85 (-OCH, dtp) \\
$24.04\left(-\mathrm{CH}_{2}, \mathrm{dtp}\right)$ \\
$136.85-129.15$ \\
$(\mathrm{Ar}-\mathrm{C})$ \\
$163.83(\mathrm{C}=\mathrm{N})$ \\
$42.19(-\mathrm{CH})$ \\
$24.62\left(-\mathrm{CH}_{2}\right)$ \\
$13.51\left(-\mathrm{CH}_{3}\right)$
\end{tabular} & 110.00 \\
\hline 10 & $\mathrm{Ti}_{2}\left(\mathrm{C}_{15} \mathrm{H}_{12} \mathrm{~N}_{2} \mathrm{OCH}_{3}\right)_{2}\left[\mathrm{~S}_{6} \mathrm{P}_{2}\left\{\mathrm{OCH}_{2} \mathrm{C}\left(\mathrm{CH}_{3}\right)_{2} \mathrm{CH}_{2} \mathrm{O}\right\}_{2}\right]$ & $\begin{array}{l}\text { 7.87-6.59(m, } 16 \mathrm{H}, \\
\mathrm{Ar}-\mathrm{H}) \\
0.97\left(\mathrm{~s}, 12 \mathrm{H},-\mathrm{CH}_{3}\right) \\
4.12\left(\mathrm{~d}, 8 \mathrm{H},-\mathrm{OCH}_{2}\right) \\
5.27(\mathrm{~s}, 4 \mathrm{H}, \mathrm{NH}) \\
3.82(\mathrm{t}, 2 \mathrm{H},-\mathrm{CH}) \\
2.15\left(\mathrm{~d}, 8 \mathrm{H},-\mathrm{CH}_{2}\right) \\
0.94\left(\mathrm{~s}, 6 \mathrm{H},-\mathrm{CH}_{3}\right)\end{array}$ & \begin{tabular}{|l|}
$22.79\left(-\mathrm{CH}_{3}, \mathrm{dtp}\right)$ \\
$31.52(\mathrm{q} \mathrm{C}, \mathrm{dtp})$ \\
$75.91 \quad\left(\mathrm{~d}, \quad-\mathrm{OCH}_{2}\right.$, \\
$\mathrm{dtp})$ \\
$43.26(-\mathrm{CH})$ \\
$165.81(\mathrm{C}=\mathrm{N})$ \\
$26.56\left(-\mathrm{CH}_{2}\right)$ \\
$136.72\left(\mathrm{Ar}^{2}-\mathrm{C}\right)$ \\
$13.56\left(-\mathrm{CH}_{3}\right)$ \\
\end{tabular} & 91.82 \\
\hline 11 & $\mathrm{Ti}_{2}\left(\mathrm{C}_{15} \mathrm{H}_{12} \mathrm{~N}_{2} \mathrm{OCH}_{3}\right)_{2}\left[\mathrm{~S}_{6} \mathrm{P}_{2}\left\{\mathrm{OC}\left(\mathrm{CH}_{3}\right)_{2} \mathrm{C}\left(\mathrm{CH}_{3}\right)_{2} \mathrm{O}\right\}_{2}\right]$ & $\begin{array}{l}1.42\left(\mathrm{~s}, 24 \mathrm{H},-\mathrm{CH}_{3}\right) \\
\text { 7.52-6.78(m, 16H, ArH }) \\
4.62(\mathrm{~s}, 4 \mathrm{H},-\mathrm{NH}) \\
3.42(\mathrm{t}, 2 \mathrm{H},-\mathrm{CH}) \\
2.19\left(\mathrm{~d}, 4 \mathrm{H},-\mathrm{CH}_{2}\right) \\
0.96\left(\mathrm{~s}, 6 \mathrm{H},-\mathrm{CH}_{3}\right)\end{array}$ & \begin{tabular}{|l|}
$26.19\left(-\mathrm{CH}_{3}, \mathrm{dtp}\right)$ \\
$92.05(\mathrm{OC}, \mathrm{dtp})$ \\
$136.76-123.16$ \\
$(\mathrm{Ar}-\mathrm{C})$ \\
$165.63(\mathrm{C}=\mathrm{N})$ \\
$45.62(-\mathrm{CH})$ \\
$25.71\left(-\mathrm{CH}_{2}\right)$ \\
$13.51\left(-\mathrm{CH}_{3}\right)$ \\
\end{tabular} & 107.62 \\
\hline 12 & $\left.\mathrm{Ti}_{2}\left(\mathrm{C}_{15} \mathrm{H}_{12} \mathrm{~N}_{2} \mathrm{OCH}_{3}\right)_{2}[\mathrm{~S}_{6} \overbrace{\mathrm{P}_{2}\left\{\mathrm{OCH}_{2} \mathrm{CH}_{2} \mathrm{CH}\left(\mathrm{CH}_{3}\right) \mathrm{O}\right.}\}_{2}\right]$ & $\begin{array}{l}4.23-3.27(\mathrm{~m}, 6 \mathrm{H}, \\
\left.-\mathrm{OCH}_{2}, \mathrm{OCH}\right) \\
2.10-1.09\left(\mathrm{~m}, 14 \mathrm{H},-\mathrm{CH}_{3},\right. \\
\left.\mathrm{CH}_{2}\right) \\
7.82-6.79(\mathrm{~m}, 16 \mathrm{H}, \mathrm{ArH}) \\
5.04(\mathrm{~s}, 4 \mathrm{H},-\mathrm{NH}) \\
2.82(\mathrm{t}, 2 \mathrm{H},-\mathrm{CH}) \\
2.02\left(\mathrm{~d}, 8 \mathrm{H},-\mathrm{CH}_{2}\right) \\
\end{array}$ & \begin{tabular}{|l|}
$136.19-123.72$ \\
$(\mathrm{Ar}-\mathrm{C})$ \\
$76.85 \quad(-\mathrm{OCH}$, \\
$\left.\mathrm{OCH}_{2}, \mathrm{dtp}\right)$ \\
$165.76(\mathrm{C}=\mathrm{N})$ \\
$13.60\left(-\mathrm{CH}_{3}\right)$ \\
$43.19(-\mathrm{CH})$ \\
$24.35\left(\mathrm{CH}_{3}, \mathrm{dtp}\right)$ \\
$27.04\left(-\mathrm{CH}_{2}\right)$ \\
\end{tabular} & 96.50 \\
\hline 13 & $\mathrm{Ti}_{2}\left(\mathrm{C}_{15} \mathrm{H}_{12} \mathrm{~N}_{2} \mathrm{O}_{2} \mathrm{CH}_{3}\right)_{2}\left[\mathrm{~S}_{6} \mathrm{P}_{2}\left(\mathrm{OCH}_{2} \mathrm{CH}_{2} \mathrm{CH}_{3}\right)_{4}\right]$ & \begin{tabular}{|l}
$7.42-6.78(\mathrm{~m}, 16 \mathrm{H}$, \\
$\mathrm{Ar}-\mathrm{H})$ \\
$1.02\left(\mathrm{t}, 12 \mathrm{H},-\mathrm{CH}_{3}\right)$ \\
$1.51\left(\mathrm{~m}, 8 \mathrm{H},-\mathrm{CH}_{2}\right)$ \\
$5.07\left(\mathrm{~m}, 8 \mathrm{H},-\mathrm{OCH}_{2}-\right)$ \\
$5.17(\mathrm{~s}, 2 \mathrm{H}, \mathrm{NH})$ \\
$3.81(\mathrm{t}, 2 \mathrm{H},-\mathrm{CH})$ \\
$2.27\left(\mathrm{~d}, 4 \mathrm{H},-\mathrm{CH}_{2}\right)$ \\
$4.29\left(\mathrm{~s}, 6 \mathrm{H},-\mathrm{OCH}_{3}\right)$ \\
\end{tabular} & - & 105.95 \\
\hline 14 & $\mathrm{Ti}_{2}\left(\mathrm{C}_{15} \mathrm{H}_{12} \mathrm{~N}_{2} \mathrm{O}_{2} \mathrm{CH}_{3}\right)_{2}\left[\mathrm{~S}_{6} \mathrm{P}_{2}\left(\mathrm{OC}_{6} \mathrm{H}_{5}\right)_{4}\right]$ & $\begin{array}{l}\text { 7.56-6.83(m, } 16 \mathrm{H}, \\
\mathrm{Ar}-\mathrm{H}) \\
7.19\left(\mathrm{~s}, 12 \mathrm{H},-\mathrm{C}_{6} \mathrm{H}_{5}\right) \\
4.78(\mathrm{~s}, 2 \mathrm{H},-\mathrm{NH}) \\
3.12(\mathrm{t}, 4 \mathrm{H},-\mathrm{CH}) \\
2.05\left(\mathrm{~d}, 8 \mathrm{H}-\mathrm{CH}_{2}\right) \\
4.00\left(\mathrm{~s}, 6 \mathrm{H},-\mathrm{CH}_{3}\right)\end{array}$ & - & 108.20 \\
\hline 15 & & $\begin{array}{l}7.68-672(\mathrm{~m}, 16 \mathrm{H}, \mathrm{Ar}-\mathrm{H}) \\
2.32-1.97\left(\mathrm{~m}, 22 \mathrm{H},-\mathrm{CH}_{3},\right. \\
\left.\mathrm{CH}_{2}\right)\end{array}$ & $\begin{array}{l}91.89 \text { (-OC, dtp) } \\
24.82 \text { (-CH, dtp) } \\
76.52 \text { (-OCH, dtp) }\end{array}$ & 95.00 \\
\hline
\end{tabular}

Bull. Chem. Soc. Ethiop. 2021, 35(1) 
Abhishek Srivastava et al.

\begin{tabular}{|c|c|c|c|c|}
\hline & $\mathrm{Ti}_{2}\left(\mathrm{C}_{15} \mathrm{H}_{12} \mathrm{~N}_{2} \mathrm{O}_{2} \mathrm{CH}_{3}\right)_{2}\left[\mathrm{~S}_{6} \mathrm{P}_{2}\left\{\mathrm{OC}\left(\mathrm{CH}_{3}\right)_{2} \mathrm{CH}_{2} \mathrm{CH}\left(\mathrm{CH}_{3}\right) \mathrm{O}\right\}_{2}\right]$ & $\begin{array}{l}4.63-4.21(\mathrm{~m}, 2 \mathrm{H},-\mathrm{OCH}) \\
5.07(\mathrm{~s}, 3 \mathrm{H},-\mathrm{NH}) \\
3.18(\mathrm{t}, 2 \mathrm{H},-\mathrm{CH}) \\
2.23\left(\mathrm{~d}, 8 \mathrm{H},-\mathrm{CH}_{2}\right) \\
4.13\left(\mathrm{~s}, 6 \mathrm{H},-\mathrm{OCH}_{3}\right)\end{array}$ & $\begin{array}{l}23.29\left(-\mathrm{CH}_{2}, \mathrm{dtp}\right) \\
136.76(\mathrm{Ar}-\mathrm{C}) \\
167.18(\mathrm{C}=\mathrm{N}) \\
43.17(-\mathrm{CH}) \\
24.18\left(-\mathrm{CH}_{2}\right) \\
57.51\left(-\mathrm{OCH}_{3}\right)\end{array}$ & \\
\hline 16 & $\mathrm{Ti}_{2}\left(\mathrm{C}_{15} \mathrm{H}_{12} \mathrm{~N}_{2} \mathrm{O}_{2} \mathrm{CH}_{3}\right)_{2}\left[\mathrm{~S}_{6} \mathrm{P}_{2}\left\{\mathrm{OCH}_{2} \mathrm{C}\left(\mathrm{CH}_{3}\right)_{2} \mathrm{CH}_{2} \mathrm{O}\right\}_{2}\right]$ & $\begin{array}{l}7.54-6.75(\mathrm{~m}, 16 \mathrm{H}, \\
\mathrm{Ar}-\mathrm{H}) \\
1.13\left(\mathrm{~s}, 12 \mathrm{H},-\mathrm{CH}_{3}\right) \\
4.08\left(\mathrm{~d}, 8 \mathrm{H},-\mathrm{OCH}_{2}-\right) \\
5.37(\mathrm{~s}, 4 \mathrm{H}, \mathrm{NH}) \\
3.16(\mathrm{t}, 2 \mathrm{H},-\mathrm{CH}) \\
2.53\left(\mathrm{~d}, 8 \mathrm{H},-\mathrm{CH}_{2}\right) \\
4.05\left(\mathrm{~s}, 6 \mathrm{H},-\mathrm{OCH}_{3}\right)\end{array}$ & $\begin{array}{l}22.83\left(\mathrm{CH}_{3}, \mathrm{dtp}\right) \\
32.02(\mathrm{q} \mathrm{C}, \mathrm{dtp}) \\
75.86 \quad\left(\mathrm{~d}, \quad-\mathrm{OCH}_{2},\right. \\
\text { dtp) } \\
137.43(\mathrm{Ar}-\mathrm{C}) \\
167.54(\mathrm{C}=\mathrm{N}) \\
42.92(\mathrm{CH}) \\
27.32\left(\mathrm{CH}_{2}\right) \\
57.29\left(-\mathrm{OCH}_{3}\right)\end{array}$ & 94.72 \\
\hline 17 & $\mathrm{Ti}_{2}\left(\mathrm{C}_{15} \mathrm{H}_{12} \mathrm{~N}_{2} \mathrm{O}_{2} \mathrm{CH}_{3}\right)_{2}\left[\mathrm{~S}_{6} \mathrm{P}_{2}\left\{\mathrm{OC}\left(\mathrm{CH}_{3}\right)_{2} \mathrm{C}\left(\mathrm{CH}_{3}\right)_{2} \mathrm{O}\right\}_{2}\right]$ & $\begin{array}{l}1.37\left(\mathrm{~s}, 24 \mathrm{H},-\mathrm{CH}_{3}\right) \\
\text { 7.63-6.81(m, } 16 \mathrm{H}, \mathrm{ArH}) \\
2.08\left(\mathrm{~d}, 4 \mathrm{H},-\mathrm{CH}_{2}\right) \\
3.41(\mathrm{t}, 2 \mathrm{H},-\mathrm{CH}) \\
4.19\left(\mathrm{~s}, 6 \mathrm{H},-\mathrm{OCH}_{3}\right) \\
5.21(\mathrm{~s}, 4 \mathrm{H},-\mathrm{NH})\end{array}$ & \begin{tabular}{|l}
$23.17\left(\mathrm{CH}_{3}, \mathrm{dtp}\right)$ \\
$90.76(\mathrm{dtp})$ \\
$42.17(\mathrm{CH})$ \\
$57.45\left(-\mathrm{OCH}_{3}\right)$ \\
$26.73\left(\mathrm{CH}_{2}\right)$ \\
$164.68(\mathrm{C}=\mathrm{N})$ \\
$136.31-122.43$ \\
$(\mathrm{~m}, \mathrm{Ar}-\mathrm{C})$
\end{tabular} & 94.06 \\
\hline 18 & $\mathrm{Ti}_{2}\left(\mathrm{C}_{15} \mathrm{H}_{12} \mathrm{~N}_{2} \mathrm{O}_{2} \mathrm{CH}_{3}\right)_{2}\left[\mathrm{~S}_{6} \mathrm{P}_{2}\left\{\mathrm{OCH}_{2} \mathrm{CH}_{2} \mathrm{CH}\left(\mathrm{CH}_{3}\right) \mathrm{O}\right\}_{2}\right]$ & $\begin{array}{l}2.69-1.29\left(\mathrm{~m}, 10 \mathrm{H},-\mathrm{CH}_{3},\right. \\
\left.\mathrm{CH}_{2}\right) \\
4.10-3.82(\mathrm{~m}, 6 \mathrm{H}, \\
-\mathrm{OCH} 2, \mathrm{OCH}) \\
7.65-6.70(\mathrm{~m}, 16 \mathrm{H}, \mathrm{ArH}) \\
5.12(\mathrm{~s}, 4 \mathrm{H},-\mathrm{NH}) \\
3.14(\mathrm{t}, 2 \mathrm{H},-\mathrm{CH}) \\
2.23\left(\mathrm{~d}, 8 \mathrm{H},-\mathrm{CH}_{2}\right) \\
4.12\left(\mathrm{~s}, 6 \mathrm{H},-\mathrm{OCH}_{3}\right)\end{array}$ & $\begin{array}{l}22.71\left(\mathrm{CH}_{3}, \mathrm{dtp}\right) \\
76.14 \quad(-\mathrm{OCH}, \\
\left.\mathrm{OCH}_{2}, \mathrm{dtp}\right) \\
42.76(\mathrm{CH}) \\
162.76(\mathrm{C}=\mathrm{N}) \\
27.92\left(\mathrm{CH}_{2}\right) \\
136.75-123.18 \\
(\mathrm{Ar}-\mathrm{C}) \\
57.21\left(-\mathrm{OCH}_{3}\right)\end{array}$ & 91.29 \\
\hline 19 & $\mathrm{Ti}_{2}\left(\mathrm{C}_{15} \mathrm{H}_{12} \mathrm{~N}_{2} \mathrm{OCl}\right)_{2}\left[\mathrm{~S}_{6} \mathrm{P}_{2}\left(\mathrm{OCH}_{2} \mathrm{CH}_{2} \mathrm{CH}_{3}\right)_{4}\right]$ & \begin{tabular}{|l}
$0.94\left(\mathrm{t}, 12 \mathrm{H},-\mathrm{CH}_{3}\right)$ \\
$7.51-6.76(\mathrm{~m}, 16 \mathrm{H}$, \\
$\mathrm{Ar}-\mathrm{H})$ \\
$2.19\left(\mathrm{~d}, 4 \mathrm{H},-\mathrm{CH}_{2}\right)$ \\
$1.61\left(\mathrm{~m}, 8 \mathrm{H},-\mathrm{CH}_{2}\right)$ \\
$(\mathrm{s}, 2 \mathrm{H}, \mathrm{NH})$ \\
$5.51\left(\mathrm{~m}, 8 \mathrm{H},-\mathrm{OCH}_{2}-\right)$ \\
$3.75(\mathrm{t}, 2 \mathrm{H},-\mathrm{CH})$
\end{tabular} & - & 106.20 \\
\hline 20 & $\mathrm{Ti}_{2}\left(\mathrm{C}_{15} \mathrm{H}_{12} \mathrm{~N}_{2} \mathrm{OCl}\right)_{2}\left[\mathrm{~S}_{6} \mathrm{P}_{2}\left(\mathrm{OC}_{6} \mathrm{H}_{5}\right)_{4}\right]$ & $\begin{array}{l}7.57-6.79(\mathrm{~m}, 16 \mathrm{H}, \\
\mathrm{Ar}-\mathrm{H}) \\
7.04\left(\mathrm{~s}, 20 \mathrm{H},-\mathrm{C}_{6} \mathrm{H}_{5}\right) \\
4.73(\mathrm{~s}, 2 \mathrm{H},-\mathrm{NH}) \\
3.09(\mathrm{t}, 4 \mathrm{H},-\mathrm{CH}) \\
2.18\left(\mathrm{~d}, 8 \mathrm{H},-\mathrm{CH}_{2}\right)\end{array}$ & - & 101.2 \\
\hline 21 & 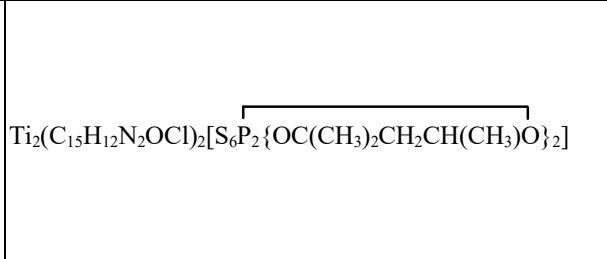 & $\begin{array}{l}7.82-6.85(\mathrm{~m}, 16 \mathrm{H}, \\
\mathrm{Ar}-\mathrm{H}) \\
4.74-4.19(\mathrm{~m}, 2 \mathrm{H},-\mathrm{OCH}) \\
2.42-1.62\left(\mathrm{~m}, 22 \mathrm{H},-\mathrm{CH}_{3},\right. \\
\left.\mathrm{CH}_{2}\right) \\
5.11(\mathrm{~s}, 2 \mathrm{H},-\mathrm{NH}) \\
3.16(\mathrm{t}, 2 \mathrm{H},-\mathrm{CH}) \\
2.27\left(\mathrm{~d}, 8 \mathrm{H}, \mathrm{CH}_{2}\right)\end{array}$ & $\begin{array}{l}91.83(-\mathrm{OC}, \mathrm{dtp}) \\
23.72(-\mathrm{CH}, \mathrm{dtp}) \\
77.35(-\mathrm{OCH}, \mathrm{dtp}) \\
24.62\left(-\mathrm{CH}_{2}, \mathrm{dtp}\right) \\
136.73(\mathrm{Ar}-\mathrm{C}) \\
167.48(\mathrm{C}=\mathrm{N}) \\
42.73(-\mathrm{CH}) \\
27.32\left(-\mathrm{CH}_{2}\right)\end{array}$ & 93.25 \\
\hline
\end{tabular}

Bull. Chem. Soc. Ethiop. 2021, 35(1) 


\begin{tabular}{|c|c|c|c|c|}
\hline 22 & $\mathrm{Ti}_{2}\left(\mathrm{C}_{15} \mathrm{H}_{12} \mathrm{~N}_{2} \mathrm{OCl}\right)_{2}\left[\mathrm{~S}_{6} \mathrm{P}_{2}\left\{\mathrm{OCH}_{2} \mathrm{C}\left(\mathrm{CH}_{3}\right)_{2} \mathrm{CH}_{2} \mathrm{O}_{2}\right]\right.$ & $\begin{array}{l}7.52-6.69(\mathrm{~m}, \mathrm{Ar}-\mathrm{H}) \\
1.10\left(\mathrm{~s}, 12 \mathrm{H},-\mathrm{CH}_{3}\right) \\
4.12\left(\mathrm{~d}, 8 \mathrm{H},-\mathrm{OCH}_{2}-\right) \\
4.79(\mathrm{~s}, 2 \mathrm{H}, \mathrm{NH}) \\
3.29(\mathrm{t}, 2 \mathrm{H},-\mathrm{CH}) \\
2.07\left(\mathrm{~d}, 8 \mathrm{H},-\mathrm{CH}_{2}\right)\end{array}$ & \begin{tabular}{|l|}
$22.63\left(\mathrm{CH}_{3}, \mathrm{dtp}\right)$ \\
$36.27(\mathrm{q} \mathrm{C}, \mathrm{dtp})$ \\
$76.32 \quad\left(\mathrm{~d}, \quad-\mathrm{OCH}_{2}\right.$, \\
$\mathrm{dtp})$ \\
$135.29(\mathrm{Ar}-\mathrm{C})$ \\
$167.76(\mathrm{C}=\mathrm{N})$ \\
$42.23(\mathrm{CH})$ \\
$26.22\left(\mathrm{CH}_{2}\right)$
\end{tabular} & 96.49 \\
\hline 23 & $\mathrm{Ti}_{2}\left(\mathrm{C}_{15} \mathrm{H}_{12} \mathrm{~N}_{2} \mathrm{OCl}\right)_{2}\left[\mathrm{~S}_{6} \mathrm{P}_{2}\left\{\mathrm{OC}\left(\mathrm{CH}_{3}\right)_{2} \mathrm{C}\left(\mathrm{CH}_{3}\right)_{2} \mathrm{O}\right\}_{2}\right]$ & $\begin{array}{l}1.42\left(\mathrm{~s}, 24 \mathrm{H},-\mathrm{CH}_{3}\right) \\
7.85-6.63(\mathrm{~m}, 16 \mathrm{H}, \mathrm{ArH}) \\
4.92(\mathrm{~s}, 2 \mathrm{H},-\mathrm{NH}) \\
3.20(\mathrm{t}, 2 \mathrm{H},-\mathrm{CH}) \\
2.13\left(\mathrm{~d}, 4 \mathrm{H},-\mathrm{CH}_{2}\right)\end{array}$ & \begin{tabular}{|l|}
$23.67\left(\mathrm{CH}_{3,} \mathrm{dtp}\right)$ \\
$91.92(\mathrm{OC}, \mathrm{dtp})$ \\
$137.81-123.47$ \\
$(\mathrm{Ar}-\mathrm{C})$ \\
$167.23(\mathrm{C}=\mathrm{N})$ \\
$43.21(\mathrm{CH})$ \\
$27.73\left(\mathrm{CH}_{2}\right)$ \\
\end{tabular} & 107.70 \\
\hline 24 & 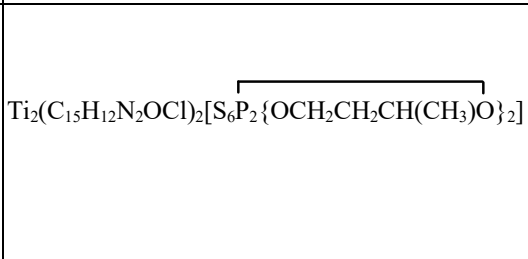 & $\begin{array}{l}2.27-1.19\left(\mathrm{~m}, 10 \mathrm{H},-\mathrm{CH}_{3} \text {, }\right. \\
\left.\mathrm{CH}_{2}\right) \\
4.09-3.82 \quad(\mathrm{~m}, \quad 6 \mathrm{H}, \quad- \\
\left.\mathrm{OCH}_{2}, \mathrm{OCH}\right) \\
7.54-6.72(\mathrm{~m}, 16 \mathrm{H}, \mathrm{ArH}) \\
5.12(\mathrm{~s}, 2 \mathrm{H},-\mathrm{NH}) \\
3.08(\mathrm{t}, 2 \mathrm{H},-\mathrm{CH}) \\
2.07\left(\mathrm{~d}, 8 \mathrm{H},-\mathrm{CH}_{2}\right)\end{array}$ & $\begin{array}{l}23.76\left(\mathrm{CH}_{3}, \mathrm{dtp}\right) \\
76.38 \quad(-\mathrm{OCH}, \\
\left.\mathrm{OCH}_{2}, \mathrm{dtp}\right) \\
43.12(\mathrm{CH}) \\
165.27(\mathrm{C}=\mathrm{N}) \\
27.35\left(\mathrm{CH}_{2}\right) \\
136.72-123.51 \\
(\mathrm{Ar}-\mathrm{C})\end{array}$ & 91.52 \\
\hline
\end{tabular}

${ }^{13}$ C NMR spectra analysis

The signals observed in the proton decoupled ${ }^{13} \mathrm{C}$ NMR spectra are in good agreement with reference to dithiophosphates and pyrazolines ligands. A complex pattern of signals detected in the region $\delta$ 136.19-123.18 ppm could be ascribed to aromatic carbon atoms. The downfield shifting of the imino carbon of $\mathrm{C}=\mathrm{N}$ from $\delta 167.45-163.78 \mathrm{ppm}$ to $\delta 143.50-142.80 \mathrm{ppm}$ (free pyrazoline) suggests that the imino nitrogen of pyrazoline is coordinated to the metal centre. The peaks due to $-\mathrm{CH}_{2}$ and $-\mathrm{CH}$ are observed at 27.72-24.62 ppm and 45.75-43.09 ppm, respectively. The peaks due to $-\mathrm{OC}$ and - $\mathrm{OCH}$ group of dithiophosphates are observed at 91.2393.09 ppm and 75.86-77.35 ppm. The NMR $\left({ }^{1} \mathrm{H},{ }^{13} \mathrm{C}\right.$ and $\left.{ }^{31} \mathrm{P}\right)$ data are summarized in Table 3 .

Table 4. FAB mass spectral data for $\mathrm{Ti}_{2}\left(\mathrm{C}_{15} \mathrm{H}_{12} \mathrm{~N}_{2} \mathrm{OH}\right)_{2}\left[\mathrm{~S}_{6} \mathrm{P}_{2}\left\{\mathrm{OCH}_{2} \mathrm{CH}_{2} \mathrm{CH}\left(\mathrm{CH}_{3}\right) \mathrm{O}\right\}_{2}\right]$.

\begin{tabular}{|c|c|c|c|c|c|c|}
\hline S.No. & Compound & $\mathrm{m} / \mathrm{z}$ & py & 2py & dtp & 2dtp \\
\hline 6 & $\mathrm{Ti}_{2}\left(\mathrm{C}_{15} \mathrm{H}_{12} \mathrm{~N}_{2} \mathrm{OH}\right)_{2}\left[\mathrm{~S}_{6} \mathrm{P}_{2}\left\{\mathrm{OCH}_{2} \mathrm{CH}_{2} \mathrm{CH}\left(\mathrm{CH}_{3}\right) \mathrm{O}\right\}_{2}\right]$ & 1059 & 882 & 585 & 375 & 163 \\
\hline 12 & $\mathrm{Ti}_{2}\left(\mathrm{C}_{15} \mathrm{H}_{12} \mathrm{~N}_{2} \mathrm{OCH}_{3}\right)_{2}\left[\mathrm{~S}_{6} \mathrm{P}_{2}\left\{\mathrm{OCH}_{2} \mathrm{CH}_{2} \mathrm{CH}\left(\mathrm{CH}_{3}\right) \mathrm{O}\right\}_{2}\right]$ & 1085 & 834 & 582 & 372 & 161 \\
\hline 18 & $\mathrm{Ti}_{2}\left(\mathrm{C}_{15} \mathrm{H}_{12} \mathrm{~N}_{2} \mathrm{O}_{2} \mathrm{CH}_{3}\right)_{2}\left[\mathrm{~S}_{6} \mathrm{P}_{2}\left\{\mathrm{OCH}_{2} \mathrm{CH}_{2} \mathrm{CH}\left(\mathrm{CH}_{3}\right) \mathrm{O}\right\}_{2}\right]$ & 1165 & 897 & 629 & 418 & 207 \\
\hline 24 & $\mathrm{Ti}_{2}\left(\mathrm{C}_{15} \mathrm{H}_{12} \mathrm{~N}_{2} \mathrm{OCl}\right)_{2}\left[\mathrm{~S}_{6} \mathrm{P}_{2}\left\{\mathrm{OCH}_{2} \mathrm{CH}_{2} \mathrm{CH}\left(\mathrm{CH}_{3}\right) \mathrm{O}\right\}_{2}\right]$ & 1124 & 893 & 582 & 371 & 160 \\
\hline
\end{tabular}

$\mathrm{dtp}=\mathrm{O}, \mathrm{O}^{\prime}$-alkylene and dialkyl dithiophosphates. $\mathrm{Py}=3$ (2'-hydroxyl phenyl)-5-(4-substituted phenyl) pyrazolines.

\section{FAB Mass spectra analysis}

The FAB mass spectra of the synthesized metal complexes have been recorded to determine the molecular weight. The molecular ion peak confirms that the metal complexes exist in dimeric 
form. FAB mass spectra of compound numbers $6,12,18$ and 24 with different substituted pyrazoline ligands in each series have been reported in Table 4.

$X R D$ and SEM studies

These crystalline/amorphous natures of the complexes have been examined through XRD. The morphology of the complexes was studied by employing XRD which shows that all the complexes are amorphous solid. The average diameter of the complexes has been calculated using "Debye Scherrer" expression (Eq. 9).

Particle size $=\mathrm{D}=0.9 \lambda / \beta \cos \theta_{\mathrm{B}}$

where, $\lambda$ is the $\mathrm{X}$-ray wavelength (1.5418 $\AA$ ), $\beta$ is corrected band broadening (full width at half maxima), $\theta_{\mathrm{B}}$ is the diffraction angle, $\mathrm{D}$ is the average nanocrystal domain diameter.

The value of full width at half maximum intensity $(\beta)$ and corresponding diffraction angle $\left(\theta_{\mathrm{B}}\right)$ is calculated using an X-ray diffractogram. The average particle size thus obtained was found to be in the range of 41-62 nm, which is further confirmed by the SEM studies (Table 5). The SEM image and $\mathrm{X}$-Ray diffractogram of $\mathrm{Ti}_{2}\left(\mathrm{C}_{15} \mathrm{H}_{12} \mathrm{~N}_{2} \mathrm{OH}\right)_{2}\left[\mathrm{~S}_{6} \mathrm{P}_{2}\left\{\mathrm{OC}\left(\mathrm{CH}_{3}\right)_{2} \mathrm{CH}_{2} \mathrm{CH}\left(\mathrm{CH}_{3}\right) \mathrm{O}\right\}_{2}\right]$ are shown in Figure 3 and Figure 4, respectively.

Table 5. Average diameter of particles determined by XRD and SEM.

\begin{tabular}{|l|c|c|c|c|}
\hline S.No. & Compound & $2 \theta$ & $\begin{array}{c}\text { Average } \\
\text { particle size } \\
(\mathrm{nm})^{*}\end{array}$ & $\begin{array}{c}\text { Average } \\
\text { particle size } \\
(\mathrm{nm})^{\dagger}\end{array}$ \\
\hline 3 & $\mathrm{Ti}_{2}\left(\mathrm{C}_{15} \mathrm{H}_{12} \mathrm{~N}_{2} \mathrm{OH}\right)_{2}\left[\mathrm{~S}_{6} \mathrm{P}_{2}\left\{\mathrm{OC}\left(\mathrm{CH}_{3}\right)_{2} \mathrm{CH}_{2} \mathrm{CH}_{\left.\left.\left(\mathrm{CH}_{3}\right) \mathrm{O}\right\}_{2}\right]}\right.\right.$ & 38.50 & 55 & 48 \\
\hline
\end{tabular}

*Determined by XRD technique. $\dagger$ Determined by SEM technique.

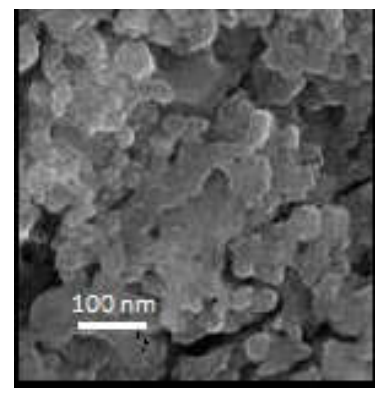

Figure 3. SEM image of $\mathrm{Ti}_{2}\left(\mathrm{C}_{15} \mathrm{H}_{12} \mathrm{~N}_{2} \mathrm{OH}\right)_{2}\left[\mathrm{~S}_{6} \mathrm{P}_{2}\left\{\mathrm{OC}\left(\mathrm{CH}_{3}\right)_{2} \mathrm{CH}_{2} \mathrm{CH}\left(\mathrm{CH}_{3}\right) \mathrm{O}\right\}_{2}\right.$. 


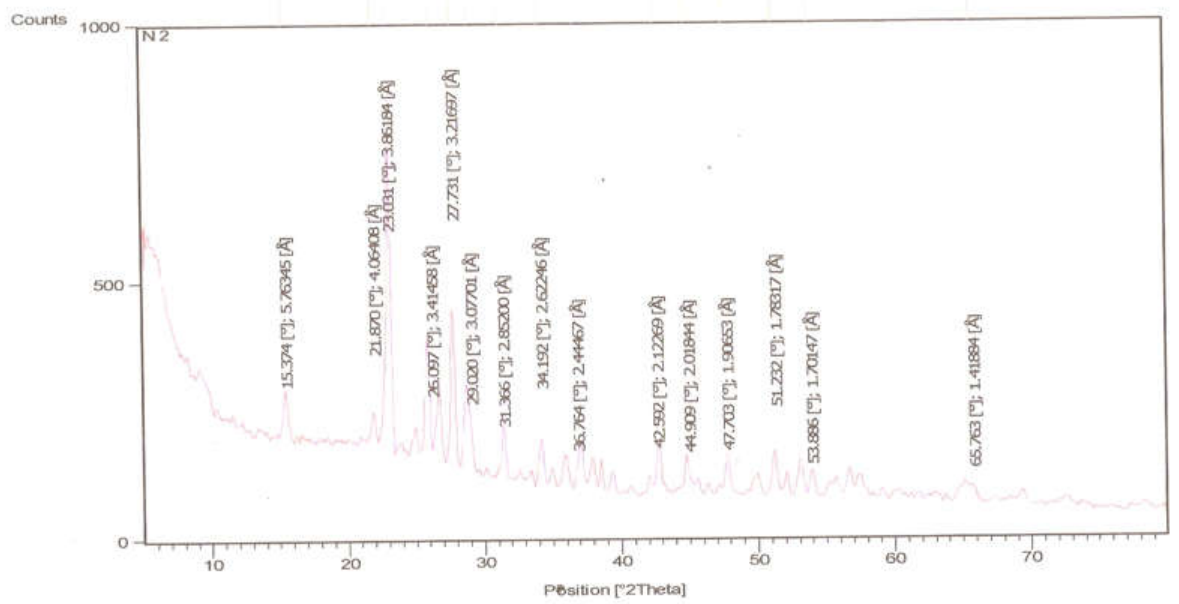

Figure 4. X-Ray diffractogram of $\mathrm{Ti}_{2}\left(\mathrm{C}_{15} \mathrm{H}_{12} \mathrm{~N}_{2} \mathrm{OH}\right)_{2}\left[\mathrm{~S}_{6} \mathrm{P}_{2}\left\{\mathrm{OC}\left(\mathrm{CH}_{3}\right)_{2} \mathrm{CH}_{2} \mathrm{CH}\left(\mathrm{CH}_{3}\right) \mathrm{O}\right\}_{2}\right.$.

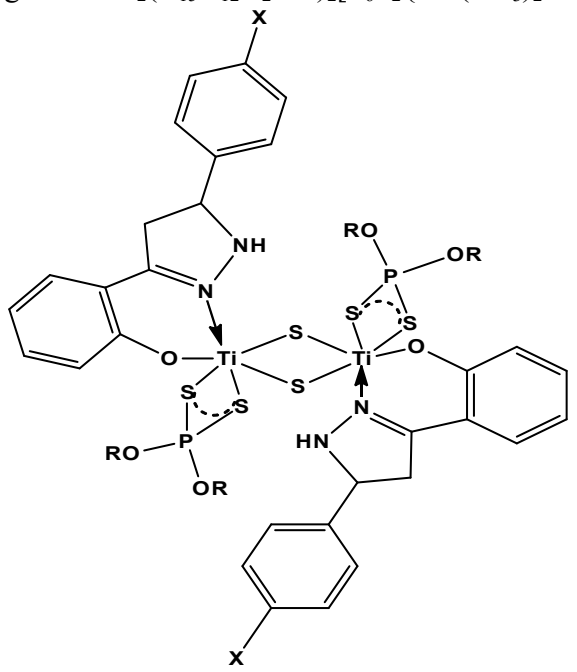

Figure 5. Proposed structure of $\left[\mathrm{Ti}_{2}\left(\mathrm{C}_{15} \mathrm{H}_{12} \mathrm{~N}_{2} \mathrm{OX}\right)_{2}(\mathrm{RO})_{4} \mathrm{P}_{2} \mathrm{~S}_{6}\right]$.

Molecular weight measurement, elemental and spectral analysis confirms the dimeric nature of the synthesized metal complexes and proposes octahedral geometry (Figure 5).

\section{CONCLUSION}

The present study describes the new route for the synthesis of Ti(IV) mixed ligand complexes with dithiophosphate and substituted pyrazoline ligands.Molecular weight measurements, elemental analysis in conjugation with spectroscopic (IR, ${ }^{1} \mathrm{HNMR},{ }^{13} \mathrm{C}$ NMR and ${ }^{31} \mathrm{P}$ NMR) studies reveal thedimeric nature of the complexes in which pyrazoline and dithiophosphate are bidentate. Scanning electron microscopic image and XRD indicate that the particles are in the nano range $(50 \mathrm{~nm})$. Coordination number six is proposed for titanium with octahedral 
geometry.This class of compound may prove to be a useful precursor for the formation of $\mathrm{TiS}_{2}$ by sulphide sol-gel due to reduced acidity of the metal centre. Further studies regarding sulphide sol-gel of these derivatives are under investigation.

\section{REFERENCES}

1. Zhang, R.; Ai, X.; Wan, Y.; Liu, Z.; Zhang, D.; Feng, S. Surface corrosion resistance in turning of titanium alloy. Int. J. Corr. 2015, 823172.

2. Schlain, D.; Kenahan, C.B. Corrosion resistance of titanium alloys compared with commercially pure titanium. Corrosion 1958, 14, 25-28.

3. Sundaram, V.J. Missile materials--current status and challenges. Bull. Mater. Sci. 1996, 19, 1025-1029.

4. Peters, M.; Kumpfert, J.; Ward, C.H.; Leyens, C. Titanium alloys for aerospace applications. Adv. Eng. Mat. 2003, 5, 419-427.

5. Muneshwar, P.; Singh, S.K.; Pant, B.; Sharma, S.C.; Mittal, M.C. Advanced processing techniques for titanium base alloys and its aluminides for space applications. Trans. Ind. Inst. Met. 2008, 61, 77-85.

6. Li, Y.; Yang, C.; Zhao, H.; Qu, S.; Li, X.; Li, Y. New developments of Ti-based alloys for biomedical applications. Materials 2014, 7, 1709-1800.

7. Prasad, K.; Bazaka, O.; Chua, M.; Rochford, M.; Fedrick, L.; Spoor, J.; Symes, R.; Tieppo, M.; Collins, C.; Cao, A.; Markwell, D.; Ostrikov, K.; Bazaka, K. Metallic biomaterials: Current challenges and opportunities. Materials 2017, 10, 884.

8. Peters, E.S.; Carmalt, C.J.; Parkins, I.P. Dual-source chemical vapour deposition of titanium sulfide thin films from tetrakisdimethylamidotitanium and sulfur precursors. J. Mater. Chem. 2004, 14, 3474-3477.

9. Carmalt, C.J.; Peters, E.S.; Parkins, I.P.; Tocher, D.A. Synthesis and characterisation of titanium pyridine- and pyrimidine-thiolates and their application as precursors to titanium disulfide. Polyhedron. 2007, 26, 43-48.

10. Corden, J.P.; Errington, W.; Moore, P.; Partridge, M.G.; Wallbridge, M.G.H. Synthesis of di-, tri- and penta-nuclear titanium(IV) species from reactions of titanium(IV) alkoxides with 2,2'-biphenol $\left(\mathrm{H}_{2} \mathrm{~L}^{1}\right)$ and 1,1'-binapthol $\left(\mathrm{H}_{2} \mathrm{~L}^{2}\right)$; crystal structures of $\left[\mathrm{Ti}_{3}\left(\mu_{2}\right.\right.$ $\left.\left.\left.\mathrm{OPr}^{\mathrm{i}}\right)_{2}\left(\mathrm{OPr}^{\mathrm{i}}\right)_{8} \mathrm{~L}^{1}\right], \quad\left[\mathrm{Ti}_{3}\left(\mathrm{OPr}^{\mathrm{i}}\right)_{6} \mathrm{~L}_{3}{ }^{1}\right], \quad\left[\mathrm{Ti}_{5}\left(\mu_{3}-\mathrm{O}\right)_{2}\left(\mu_{2}-\mathrm{OR}\right)_{2}(\mathrm{OR})_{6} \mathrm{~L}_{4}^{1}\right)\right] \quad\left(\mathrm{R}=\mathrm{OPr}^{\mathrm{i}}, \quad \mathrm{OBu}^{\mathrm{n}}\right) \quad$ and $\left[\mathrm{Ti}_{2}\left(\mathrm{OPr}^{\mathrm{i}}\right)_{4} \mathrm{~L}_{2}^{2}\right]$. Dalton Trans. 2004, 1846-1851.

11. Uppal, R.; Incarvito, C.D.; Lakshmi, K.V.; Valentine, A.M. Aqueous spectroscopy and redox properties of carboxylate-bound titanium. Inorg. Chem. 2006, 45, 1795-1804.

12. Baranwal, B.P.; Fatima, T.; Singh, A.K.; Varma, A. Nano-sized titanium(IV) ternary and quaternary complexes with electron-rich oxygen-based bidentate ligand. Inorg. Chim. Acta 2009, 362, 3461-3464.

13. Blower, P.J.; Dilworth, J.R. Thiolato-complexes of the transition metals. Coord. Chem. Rev. 1987, 76, 121-185.

14. Drew, M.G.B.; Forsyth, G.A.; Hasan, M.; Hobson, R.J.; Rice, D.A. Reactions of copper(II) with $\left[\mathrm{S}_{2} \mathrm{P}(\mathrm{OR})_{2}\right]^{-}\left(\mathrm{R}=\mathrm{Et}\right.$ or $\left.\mathrm{Pr}^{\mathrm{i}}\right)$ and single-crystal X-ray studies of $\mathrm{Cu}_{2}\left[\mathrm{~S}_{2} \mathrm{P}(\mathrm{OEt})_{2}\right]$-bipy and $\mathrm{Cu}\left[\mathrm{S}_{2} \mathrm{P}(\mathrm{OEt})_{2}\right] \cdot 2 \mathrm{PPh}_{3}$.J. Chem. Soc. Dalton. Trans. 1987, 1027-1033.

15. Srivastava, A.; Srivastava, N.; Tripathi, U.N.; Siddiqui, A. Synthesis and characterization of mixed ligand complexes of zirconium(IV) with sulphur, nitrogen and oxygen donor ligands. Chem. Chem. Technol. 2019, 13, 23-32.

16. Cazaux, J.N.; Braunstein, P.; Magna, L.; Saussine, L.; Bourbigou, H.O. Mono-(aryloxido) titanium(IV) complexes and their application in the selective dimerization of ethylene. Eur. J. Inorg. Chem. 2009, 20, 2942-2950. 
17. Bae, D.Y.; Park, G.S.; Ko, N.; Son, K.; Lee, E. First-row early transition metal complexes with a highly sterically demanding triisopropylphenyl amino triphenolate ligand: Synthesis and applications. Dalton Trans. 2019, 48, 9617-9624.

18. Li, X.; Yang, B.; Zheng, H.; Wu, P.; Zeng, G. Synthesis and characterization of salen-Ti(IV) complex and application in the controllable polymerization of D,L-lactide. PLoS. One 2018, 13, e0201054.

19. Shin, H.; Park, Y.; Kim, S.; An, B-K.; Park, J. Synthesis and characterization of titanium complex with a dithiolate ligand for green LCD color filter dyes. Nanoscale Res. Lett. 2012, 7, 635 .

20. Menendez, E. Titanium complexes in cancer treatment. Crit. Rev. Oncol. Hemat. 2002, 42, 309-315.

21. Kaushal, R.; Kumar, N.; Chaudhary, A.; Arora, S.; Awasthi, P. Synthesis, spectral characterization, and antiproliferative studies of mixed ligand titanium complexes of adamantylamine. Bioinorg. Chem. Appl. 2014, Article ID 142828.

22. Miller, M.; Tshuva, E.Y. Synthesis of pure enantiomers of titanium(IV) complexes with chiral diaminobis (phenolato) ligands and their biological reactivity. Sci. Rep. 2018, 8, 9705.

23. Kaushal, R.; Kumar, N.; Thakur, A.; Nehra, K.; Awasthi, P.; Kaushal, R.; Arora, S. Synthesis, spectral characterization, antibacterial and anticancer activity of some titanium complexes. Anticancer Agents Med. Chem. 2018, 18, 739-746.

24. Kumar, N.; Kaushal, R.; Chaudhary, A.; Arora, S.; Awasthi, P. Titanium based mixed ligand complexes: Synthesis, spectroscopic and in vitro antiproliferative studies. Inorg. Nano-Met. Chem. 2018, 48, 467-476.

25. Stephan, D.W.; Nadasdi, T.T. Early transition metal thiolates. Coord. Chem. Rev. 1996, 146, 147-150.

26. Tilmann, G.K.; Karlheinz, S. Metalation studies on titanocene dithiolates. Inorganics 2018 , 6,85 .

27. Kumar, A.; Chauhan, R.; Molloy, K.; Kociok-Köhn, G.; Bahadur, L.; Singh, N. Synthesis, structure and light-harvesting properties of some new transition-metal dithiocarbamates involving ferrocene. Chem. Eur. J. 2010, 16. 4307-4314.

28. Donzelli, A.; Potvin, P.G. Dithiocarbamate complexes of Ti(IV) alkoxides: Synthesis, characterization, and electrochemistry. Inorg. Chem. 2009, 48, 9, 4171-4178.

29. Matsuzaki, K.; Kawaguchi, H.; Voth, P.; Noda, K.; Itoh, S.; Takagi, H.D.; Kashiwabara, K.; Tatsumi, K. Syntheses and characterization of titanium(IV) and titanium(III) complexes with (2-dimethylphosphino)ethane-1-thiolate and (3-dimethylphosphino) propane-1-thiolate as ligands. Inorg. Chem. 2003, 42, 5320-5329.

30. Tripathi, U.N.; Siddiqui, A.; Safi, M.; Sharma, N.; Srivastava, N. Synthesis, characterization and sol-gel behavior of dichlorotitanium(IV) (O-alkyl, O-cycloalkyl and O-aryl trithiophosphates). Phosphorus Sulfur Silicon Relat. Elem. 2010, 185, 1993-2001.

31. Tripathi, U.N.; Srivastava, N.; Ahmed, M.S.; Siddiqui, A.; Dwivedi, S.K. Synthesis and spectral behavior of nanometric Ti(IV) complexes with nitrogen, sulphur and oxygen donors. J. Coord. Chem. 2011, 64, 3938-3949.

32. Mayoral, V.; Tian, R.; Kelly, A.G.; Griffin, A.; Harvey, A.; Borrelli, M.; Nisi, K.; Backes, C.; Coleman, J.N. Solvent exfoliation stabilizes $\mathrm{TiS}_{2}$ nanosheets against oxidation, facilitating lithium storage applications. Nanoscale 2019, 11, 6206-6216.

33. Licklederer, M.; Cha, G.; Hahn, R.; Schmuki, P. Ordered nanotubular titanium disulfide $\left(\mathrm{TiS}_{2}\right)$ structures: Synthesis and use as counter electrodes in dye sensitized solar cells (DSSCs). J. Electrochem. Soc. 2019, 166, 3009-3013

34. Parvaz, M.; Ahmed, S.; Khan, M.B.; Khan, Z.H. Synthesis of $\mathrm{TiS}_{2}$ nano-discs for supercapacitor application. AIP Conference Proceedings 2018, 1953, 030121. 
35. Tao, H.; Zhou, M.; Wang, R.; Wang, K.; Chen, S.; Jiang, K. TiS ${ }_{2}$ as an advanced conversion electrode for sodium-ion batteries with ultrahigh capacity and long cycle life. Adv. Sci. 2018, 5,1801021

36. Vogel, A. A Text Book of Quantitative Inorganic Analysis, ELBS Longman Group Ltd: London; 1998.

37. Zhang, Z.; Tan, Y.-J.; Wang, C.-S.; Wu, H.-H. One-pot synthesisof 3,5-diphenyl-1Hpyrazoles from chalcones and hydrazine under mechanochemical ball milling. Heterocycles 2014, 89, 103-112.

38. Chauhan, H.P.S.; Chauhan, B.P.S.; Srivastava, G.; Metrotra, R.C. Synthesis and characterization of dioxaphospholanes and dioxaphosphorinanes. Phosphorus Sulphur Silicon 1983, 15, 99-104.

39. Tripathi, U.N.; Solanki, J.S.; Ahmed, M.S.; Bhardwaj, A. Synthesis, spectral and antimicrobial studies of chloroantimony(III)di[3(2'-hydroxyphenyl)-5-(4-substituted phenyl pyrazolinates]. J. Coord. Chem. 2009, 62, 636-644.

40. Sharma, K.V.; Sharma, V.; Tripathi, U.N. Iron(III)5(2'-hydroxyphenyl)-3-(4-X-phenyl) pyrazolinates and their addition complexes with N, P donor ligands: Synthesis, spectral and antimicrobial investigations. J. Coord. Chem. 2008, 61, 3314-3328.

41. Quin, L.D. The heterocyclic Chemistry of Phosphorus, Wiley Interscience: New York; 1981.

42. Thomas, L.C. Interpretation of the IR spectra of Organophosphorus Compounds, Heyden: London; 1974.

43. Tripathi, U.N.; Bipin, P.P.; Mirza, R.; Chaturvedi, A. Synthesis and spectroscopic characterization of addition complexes of copper dithiophosphates with heterocyclic nitrogen donor bases. Pol. J. Chem. 1999, 73, 1751-1756.

44. Tripathi, U.N.; Bipin, P.P.; Mirza, R.; Shukla, S. Synthesis and characterization of O,O' dialkyl and alkylene dithiophosphates of lanthanum(III) and their adducts with nitrogen and phosphorus donor bases. J. Coord. Chem. 2002, 55, 1111-1118.

45. Grayson, M.; Griffith, E.J. (Eds.). Topics in Phosphorus Chemistry, Vol. 5, Wiley Interscience: New York; 1967.

46. Silverstein, R.M.; Wbster, F.X. Spectrometric Identification of Organic Compounds, Wiley Interscience: New York; 1998.

47. Nakamoto, K. IR Spectra of Inorganic and Coordination Compounds, Wiley Interscience: New York; 1970.

48. Glidewell, C. Ambident nucleophiles: VI. Solution metal-ligand binding modes in phosphorodithioate complexes. A phosphorus-31 N.M.R. study. Inorg. Chem. Acta 1977, $25,159-163$. 